\title{
Research Article \\ Comparative Analysis of Mathematical Models for Blood Flow in Tapered Constricted Arteries
}

\author{
D. S. Sankar ${ }^{1}$ and Yazariah Yatim ${ }^{2}$ \\ ${ }^{1}$ Division of Mathematics, School of Advanced Sciences, VIT University, Chennai Campus, \\ Chennai 600 127, India \\ ${ }^{2}$ School of Mathematical Sciences, Universiti Sains Malaysia, 11800 Penang, Malaysia \\ Correspondence should be addressed to D.S. Sankar, sankar_ds@yahoo.co.in
}

Received 27 February 2012; Accepted 19 July 2012

Academic Editor: Gaston Mandata N'Guerekata

Copyright (C) 2012 D. S. Sankar and Y. Yatim. This is an open access article distributed under the Creative Commons Attribution License, which permits unrestricted use, distribution, and reproduction in any medium, provided the original work is properly cited.

\begin{abstract}
Pulsatile flow of blood in narrow tapered arteries with mild overlapping stenosis in the presence of periodic body acceleration is analyzed mathematically, treating it as two-fluid model with the suspension of all the erythrocytes in the core region as non-Newtonian fluid with yield stress and the plasma in the peripheral layer region as Newtonian. The non-Newtonian fluid with yield stress in the core region is assumed as (i) Herschel-Bulkley fluid and (ii) Casson fluid. The expressions for the shear stress, velocity, flow rate, wall shear stress, plug core radius, and longitudinal impedance to flow obtained by Sankar (2010) for two-fluid Herschel-Bulkley model and Sankar and Lee (2011) for two-fluid Casson model are used to compute the data for comparing these fluid models. It is observed that the plug core radius, wall shear stress, and longitudinal impedance to flow are lower for the two-fluid H-B model compared to the corresponding flow quantities of the two-fluid Casson model. It is noted that the plug core radius and longitudinal impedance to flow increases with the increase of the maximum depth of the stenosis. The mean velocity and mean flow rate of two-fluid H-B model are higher than those of the two-fluid Casson model.
\end{abstract}

\section{Introduction}

Atherosclerosis is an arterial disease in humans, which leads to the malfunctioning of the cardiovascular system [1]. The intimal thickening of an artery is the initial stage in the progression of atherosclerosis [2-4]. The lumen of the arteries is narrowed by the development of atherosclerotic plaques that protrude into the lumen, resulting in stenosed arteries. The wall of the artery is stiffened by the growth of plaque with a lipid core and a fibromuscular cap and narrowing of lumen of the artery by the deposit of fats, lipids, cholesterol, and so forth [5]. Stenoses in different shapes are formed in the arterial lumen 
and some of the stenoses shape are axisymmetric, asymmetric, overlapping, and multiple $[1,6-8]$. When a stenosis is developed in an artery, its serious consequences are the increased resistance and the associated reduction of blood flow in the downstream $[9,10]$. Thus, the development of a stenosis in the lumen of an artery leads to the serious circulatory disorder. Chakravarty et al. [11] pointed out that the blood vessels bifurcate at frequent intervals and although the individual segments of arteries may be treated as uniform between bifurcations, the diameter of the artery reduces considerably at each bifurcation. How and Black [12] pronounced that the study of blood flow in tapered arteries is useful in the design of prosthetic blood vessels as the use of grafts of tapered lumen has the surgical advantage. Hence, it is important to mathematically analyze the blood flow in tapered arteries with stenosis.

In many situations of our routine life such as traveling in vehicles, aircrafts, ships, swinging in a cradle, subjecting to vibration therapy as a treatment for some disease, sudden movements of body in sports activities, our body is exposed to body accelerations or vibrations $[8,13-15]$. In some situations like traveling in a bus/train, the whole of the body is subjected to vibrations, while in some other occasions such as when operating jack hammer or lathe machine, driving a car, applying vibration therapy as a medical treatment, some specific part of our body is forced to vibrations [16, 17]. Exposure of our body to high level unintended external body accelerations for a long period causes serious health hazards due to the abnormal functioning of the cardiovascular system [18], and this leads to serious cardiovascular diseases which show some symptoms like headache, abdominal pain, increase in pulse rate, venous pooling of blood in the extremities, loss of vision, and hemorrhage in the face, neck, eye sockets, lungs, and brain [16, 18-20]. Thus, it is useful to investigate the effect of periodic body accelerations on the physiologically important flow measurements of blood flow in arteries of different diameters.

Blood exhibits anomalous viscous properties. Blood, when it flows in larger diameter arteries at high shear rates, it behaves like Newtonian fluid, but when it flows through narrow diameter arteries at low shear rates, it shows notable non-Newtonian behavior [21]. Several researchers investigated blood flow properties in constricted narrow arteries in the absence and presence of externally imposed periodic body accelerations [22-27].

Several researchers $[11,28,29]$ mentioned that when blood flows in smaller diameter blood vessels at low shear rates, there is erythrocyte-free plasma layer adjacent to the vessel wall and core layer of suspension of all erythrocytes and thus it is not realistic to model blood as simply a single fluid non-Newtonian model. Hence, it is appropriate to model blood as a two-fluid model when it flows through narrow diameter arteries at low shear rates (diameter up to $1300 \mu \mathrm{m}$ ) [30], treating the suspension of all the erythrocytes in the core region as a nonNewtonian fluid and the cell free plasma in the peripheral layer region as Newtonian fluid. Herschel-Bulkley (H-B) fluid model and Casson fluid model are some of the non-Newtonian fluid models with yield stress which are commonly used as the non-Newtonian fluids to represent the suspension of all the erythrocytes in the core region of blood flow in narrow arteries [21, 28]. Some advantages of using H-B fluid rather than Casson fluid to model the suspension of all the erythrocytes in the core region of the two-fluid flow modeling of blood in narrow arteries are mentioned below.

Iida [31] reports "the velocity profiles of blood when it flows in the arterioles having diameter less than $0.1 \mathrm{~mm}$ are generally explained fairly by both Casson and H-B fluid models. However, the velocity profiles of blood flow in the arterioles whose diameters are less than $0.065 \mathrm{~mm}$ do not conform to the Casson fluid, but can still be explained by H-B fluid." $\mathrm{Tu}$ and Deville [22] reported that blood obeys Casson fluid's constitutive equation only at 
moderate shear rates, whereas H-B fluid's constitutive equation can be used still at low shear rates and represents fairly closely what is occurring in blood. Chaturani and Palanisamy [6] propounded that when blood flows in arteries of diameter $0.095 \mathrm{~mm}$, it behaves like H-B fluid rather than other non-Newtonian fluids. Moreover, Casson fluid's constitutive equation has only one parameter namely the yield stress, whereas the H-B fluid's constitutive equation has one more parameter, namely, the power law index " $n$ ", and thus one can obtain more detailed information about the blood flow characteristics by using the H-B fluid model rather than Casson fluid model [32]. Hence, it is appropriate to represent the suspension of all the erythrocytes in the core region of the two-fluid model of blood (when it flows in narrow diameter arteries at low shear rates) by H-B fluid rather than Casson fluid.

Sankar [33] and Sankar and Lee [34] studied the two-fluid H-B model and twofluid Casson model, respectively, for blood flow in a narrow artery with mild axisymmetric stenosis under body accelerations. The pulsatile flow of two-fluid H-B fluid model and two-fluid Casson fluid model for blood flow through narrow tapered arteries with mild overlapping stenosis under periodic body acceleration has not been studied so far, to the knowledge of the authors. Hence, in this study, a comparative study is performed for the pulsatile flow of two-fluid H-B and Casson models for blood flow in narrow tapered arteries with mild overlapping stenoses in the presence of periodic body acceleration. For the two-fluid H-B model, the expressions obtained in Sankar [33] for shear stress, velocity distribution, wall shear stress, and flow rate are used to compute the data for the present comparative study. The aforesaid flow quantities obtained by Sankar and Lee [34] for twofluid Casson model are also used to compute the data for this comparative study. The layout of the paper is as follows.

Section 2 mathematically formulates the two-fluid H-B and Casson models for blood flow and applies the perturbation method of solution. In Section 3, the results of two-fluid H-B model and two-fluid Casson model for blood flow in narrow tapered arteries with mild overlapping stenosis are compared. Some possible clinical applications to the present study are also given in Section 3. The main results are summarized in the concluding Section 4.

\section{Mathematical Formulation}

Consider an axially symmetric, laminar, pulsatile, and fully developed flow of blood (assumed to be incompressible) in the axial $(\bar{z})$ direction through a narrow tapered artery with mild overlapping stenosis. Geometry of the segment of a narrow artery with mild overlapping stenosis is shown in Figure 1(a). For different angles of tapering, the geometry of the stenosed artery is depicted in Figure 1(b). The geometry of the stenosed tapered artery at a cross-section in a time cycle is sketched in Figure 1(c). The segment of the artery under study is considered to be long enough so that the entrance, end, and special wall effects can be neglected. Since, the stenosis developed in the lumen of the segment of artery, it is appropriate to treat the segment of the stenosed artery under study as rigid walled. Assume that there is periodical body acceleration in the region of blood flow. Blood is modeled as a two-fluid model, treating the suspension of all the erythrocytes in the core region as non-Newtonian fluid with yield stress and the plasma in the peripheral layer region as Newtonian fluid. The non-Newtonian fluid in the core region is represented by (i) Herschel-Bulkley $(\mathrm{H}-\mathrm{B})$ fluid model and (ii) Casson fluid model. Cylindrical polar coordinate system $(\bar{r}, \bar{\psi}, \bar{z})$ is used to analyze the blood flow. 


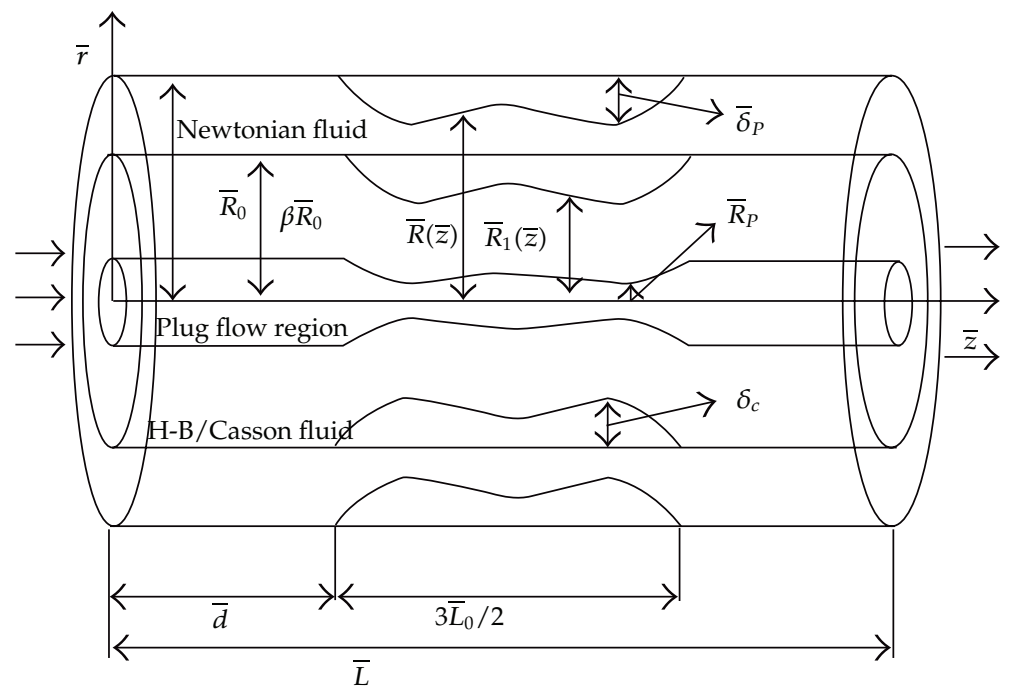

(a) Geometry of the segment of an artery with overlapping stenosis

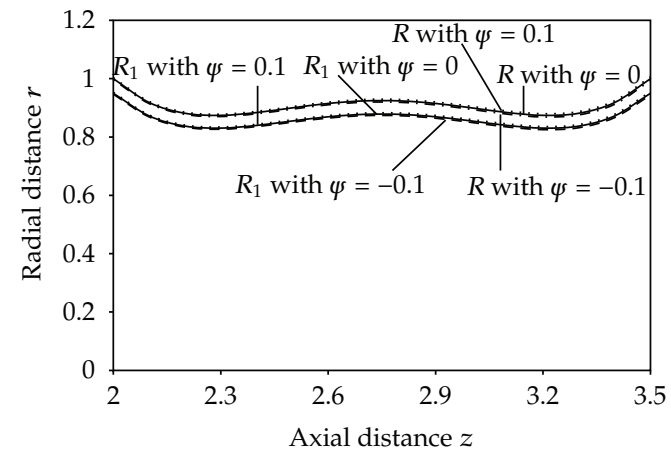

(b) Shapes of the overlapping stenosis in the peripheral layer and core regions for different angles of tapering at $t=0^{\circ}$

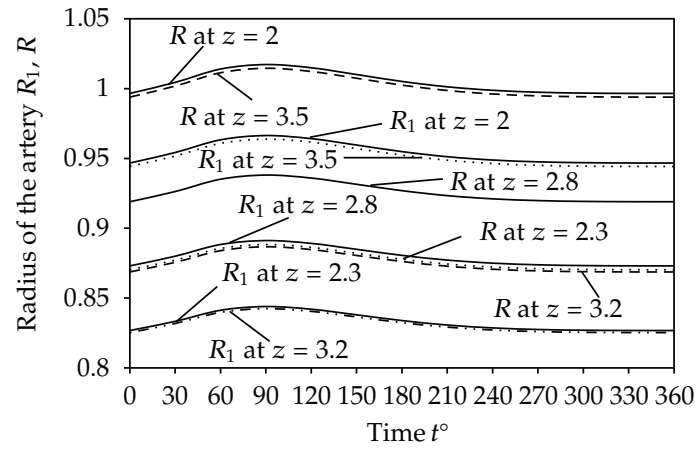

(c) Changes in the shape of the arterial geometry in a time cycle at $z=2.3$ and $\psi=-0.1$

Figure 1: Pictorial description of segment of the artery with overlapping stenosis.

\subsection{Two-Fluid Herschel-Bulkley (H-B) Model}

\subsubsection{Governing Equations and Boundary Conditions}

The geometry of the artery as shown in Figure 1 is mathematically defined as follows [29, 35]:

$$
\begin{aligned}
& \left(\bar{R}, \bar{R}_{1}\right)(\bar{z}, \bar{t}) \\
& \quad= \begin{cases}{\left[\left(m \bar{z}+\bar{r}_{0}\right)(1, \alpha)-\left(\bar{\delta}_{P}, \bar{\delta}_{C}\right)\left(\frac{\cos \psi}{\bar{L}_{0}}\right)(\bar{z}-\bar{d}) \bar{g}(\bar{z})\right] \bar{a}_{1}(\bar{t})} & \text { if } \bar{d} \leq \bar{z} \leq \bar{d}+\left(\frac{3 \bar{L}_{0}}{2}\right), \\
(1, \alpha)\left(m \bar{z}+\bar{r}_{0}\right) \bar{a}_{1}(\bar{t}) & \text { otherwise, }\end{cases}
\end{aligned}
$$


where

$$
\begin{gathered}
\bar{g}(\bar{z})=\left\{11-\left(\frac{94}{3 \bar{L}_{0}}\right)(\bar{z}-\bar{d})+\left(\frac{32}{\bar{L}_{0}^{2}}\right)(\bar{z}-\bar{d})^{2}-\left(\frac{32}{3 \bar{L}_{0}^{3}}\right)(\bar{z}-\bar{d})^{3}\right\}, \\
\bar{a}_{1}(\bar{t})=1-b\left(\cos \bar{\omega}_{P} \bar{t}-1\right) e^{-b \bar{\omega}_{p} \bar{t}}
\end{gathered}
$$

where $\bar{R}(\bar{z}, \bar{t}), \bar{R}_{1}(\bar{z}, \bar{t})$ are the radius of the tapered stenosed arterial segment in the peripheral layer region and core region, respectively; $\bar{r}_{0}$ is the radius of the artery in the normal region; $\psi$ and $m(=\tan \psi)$ are the angle of tapering and slope of the tapered vessel respectively; $d$ is the location of the stenosis; $3 \bar{L}_{0} / 2$ is the length of the stenosis; $\bar{\delta}_{P} \cos \psi$, $\bar{\delta}_{C} \cos \psi$ are the critical heights of the overlapping stenosis in the peripheral layer region and core region, respectively; $\bar{\delta}_{C}=\alpha \bar{\delta}_{P}, \bar{a}_{1}(\bar{t})$ is the time variant parameter; $b$ is a constant; $\bar{\omega}\left(=2 \pi \bar{f}_{P}\right)$ is the angular frequency with $\bar{f}_{P}$ as the pulse frequency. Length of the arterial segment is taken to be of finite length $\bar{L}$. It has been reported that the radial velocity is negligibly small and can be neglected for a low Reynolds number flow in a narrow artery with mild stenosis. The momentum equations governing the blood flow in the axial and radial directions simplify respectively to [33] as follows:

$$
\begin{gathered}
\bar{\rho}_{H} \frac{\partial \bar{u}_{H}}{\partial \bar{t}}=-\frac{\partial \bar{p}}{\partial \bar{z}}-\frac{1}{\bar{r}} \frac{\partial}{\partial \bar{r}}\left(\bar{r}_{H}\right)+\bar{F}(\bar{t}) \quad \text { in } 0 \leq \bar{r} \leq \bar{R}_{1}(\bar{z}) \\
\bar{\rho}_{N} \frac{\partial \bar{u}_{N}}{\partial \bar{t}}=-\frac{\partial \bar{p}}{\partial \bar{z}}-\frac{1}{\bar{r}} \frac{\partial}{\partial \bar{r}}\left(\overline{r \tau}_{N}\right)+\bar{F}(\bar{t}) \quad \text { in } \bar{R}_{1}(\bar{z}) \leq \bar{r} \leq \bar{R}(\bar{z}) \\
0=-\frac{\partial \bar{p}}{\partial \bar{r}^{\prime}}
\end{gathered}
$$

where the shear stress $\bar{\tau}=\left|\bar{\tau}_{\overline{r z}}\right|=-\bar{\tau}_{\overline{r z}}$ (since $\bar{\tau}=\bar{\tau}_{H}$ or $\bar{\tau}=\bar{\tau}_{N}$ ). The constitutive equations of the fluids in motion in the core region (for H-B fluid) and in the peripheral region (for Newtonian fluid) are given by

$$
\begin{gathered}
\bar{\tau}_{H}=\sqrt[n]{\bar{\mu}_{H}\left(\frac{\partial \bar{u}_{H}}{\partial \bar{r}}\right)}+\bar{\tau}_{y} \quad \text { if } \bar{\tau}_{H} \geq \bar{\tau}_{y}, \bar{R}_{p} \leq \bar{r} \leq \bar{R}_{1}(\bar{z}) \\
\frac{\partial \bar{u}_{H}}{\partial \bar{r}}=0 \quad \text { if } \bar{\tau}_{H} \leq \bar{\tau}_{y}, 0 \leq \bar{r} \leq \bar{R}_{p} \\
\bar{\tau}_{N}=\bar{\mu}_{N}\left(-\frac{\partial \bar{u}_{N}}{\partial \bar{r}}\right) \quad \text { if } \bar{R}_{1}(\bar{z}) \leq \bar{r} \leq \bar{R}(\bar{z})
\end{gathered}
$$

where $\bar{u}_{H}, \bar{u}_{N}$ are the axial component of the fluid's velocity in the core region and peripheral region; $\bar{\tau}_{H}, \bar{\tau}_{N}$ are the shear stress of the fluid in the core region (H-B fluid) and peripheral layer region (Newtonian fluid); $\bar{\mu}_{H}, \bar{\mu}_{N}$ are the viscosities of the H-B fluid and Newtonian fluid with respective dimensions $\left[M L^{-1} T^{-2}\right]^{n} T$ and $M L^{-1} T^{-1} ; \bar{\rho}_{H}, \bar{\rho}_{N}$ are the densities of the $\mathrm{H}-\mathrm{B}$ fluid and Newtonian fluid; $\bar{p}$ is the pressure; $\bar{t}$ is the time; $\bar{\tau}_{y}$ is the yield stress of the fluid in the core region. From (2.6), it is clear that the velocity gradient vanishes in the region where 
the shear stress is less than the yield stress which implies a plug flow whenever $\bar{\tau}_{H} \leq \bar{\tau}_{y}$ and normal flow otherwise. The boundary conditions are

$$
\begin{gathered}
\bar{\tau}_{H} \text { is finite, } \frac{\partial \bar{u}_{H}}{\partial \bar{r}}=0 \quad \text { at } \bar{r}=0, \\
\bar{\tau}_{H}=\bar{\tau}_{N} \quad \text { at } \bar{r}=\bar{R}_{1}(\bar{z}), \\
\bar{u}_{H}=\bar{u}_{N} \quad \text { at } \bar{r}=\bar{R}_{1}(\bar{z}), \\
\bar{u}_{N}=0 \quad \text { at } \bar{r}=\bar{R}(\bar{z}) .
\end{gathered}
$$

Since the blood flow in arteries is due to the applied pressure gradient (due to the pumping action of the heart) and is highly pulsatile, it is appropriate to assume the pressure gradient as the following periodic function of $\bar{z}$ and $\bar{t}[16,20]$.

$$
-\frac{\partial \bar{p}}{\partial \bar{z}}(\bar{z}, \bar{t})=A_{0}+A_{1} \cos \left(\bar{\omega}_{p} \bar{t}\right)
$$

where $A_{0}$ is the steady component of the pressure gradient, $A_{1}$ is the amplitude of the pulsatile component of the pressure gradient, and $\bar{\omega}_{p}=2 \pi \bar{f}_{p^{\prime}} \bar{f}_{p}$ is the pulse frequency in Hz. Both $A_{0}$ and $A_{1}$ are functions of $\bar{z}$ [16]. The periodic body acceleration in the axial direction is given by

$$
\bar{F}(\bar{t})=a_{0} \cos \left(\bar{\omega}_{b} \bar{t}+\phi\right)
$$

where $a_{0}$ is the amplitude, $\bar{\omega}_{b}=2 \pi \bar{f}_{b}, \bar{f}_{b}$ is the frequency in Hz and is assumed to be small so that the wave effect can be neglected [20], and $\phi$ is the lead angle of $\bar{F}(\bar{t})$ with respect to the heart action.

\subsubsection{Nondimensionalization}

Let us introduce the following nondimensional variables:

$$
\begin{gathered}
z=\frac{\bar{z}}{\bar{R}_{0}}, \quad R(z)=\frac{\bar{R}(\bar{z})}{\bar{R}_{0}}, \quad R_{1}(z)=\frac{\bar{R}_{1}(\bar{z})}{\bar{R}_{0}}, \quad r=\frac{\bar{r}}{\bar{R}_{0}}, \quad t=\bar{\omega}_{P} \bar{t}, \\
d=\frac{\bar{d}}{\bar{R}_{0}}, \quad L_{0}=\frac{\bar{L}_{0}}{\bar{R}_{0}}, \quad \omega=\frac{\bar{\omega}_{b}}{\bar{\omega}_{P}}, \quad u_{H}=\frac{\bar{u}_{H}}{A_{0} \bar{R}_{0}^{2} / 4 \bar{\mu}_{0}}, \quad u_{N}=\frac{\bar{u}_{N}}{A_{0} \bar{R}_{0}^{2} / 4 \bar{\mu}_{N}}, \\
\tau_{H}=\frac{\bar{\tau}_{H}}{A_{0} \bar{R}_{0} / 2}, \quad \tau_{N}=\frac{\bar{\tau}_{N}}{A_{0} \bar{R}_{0} / 2}, \quad \theta=\frac{\bar{\tau}_{y}}{A_{0} \bar{R}_{0} / 2}, \quad \alpha_{H}^{2}=\frac{\bar{R}_{0}^{2} \overline{\omega \rho}_{H}}{\bar{\mu}_{0}}, \\
\alpha_{N}^{2}=\frac{\bar{R}_{0}^{2} \overline{\omega \rho}_{N}}{\bar{\mu}_{N}}, \quad R_{p}=\frac{\bar{R}_{p}}{\bar{R}_{0}}, \quad \delta_{p}=\frac{\bar{\delta}_{p}}{\bar{R}_{0}}, \quad \delta_{C}=\frac{\bar{\delta}_{C}}{\bar{R}_{0}}, \quad e=\frac{A_{1}}{A_{0}}, \quad B=\frac{a_{0}}{A_{0}},
\end{gathered}
$$


where $\bar{\mu}_{0}=\bar{\mu}_{H}\left(2 / A_{0} \bar{R}_{0}\right)^{n-1}$, which has the dimension as that of the Newtonian fluid's viscosity, $\alpha_{H}$ is the pulsatile Reynolds number or generalized Wormersly frequency parameter, and when $n=1$, we get the Wormersly frequency parameter $\alpha_{N}$ of the Newtonian fluid. Applying (2.11) into (2.1)-(2.2), one can get the nondimensional form of the equations for the geometry of the tapered stenosed arterial segment as follows:

$$
\begin{aligned}
& \left(R, R_{1}\right)(z, t) \\
& \quad= \begin{cases}{\left[\left(m z+r_{0}\right)(1, \alpha)-\left(\delta_{P}, \delta_{C}\right)\left(\frac{\cos \psi}{L_{0}}\right)(z-d) g(z)\right] a_{1}(t)} & \text { if } d \leq z \leq d+\left(\frac{3 L_{0}}{2}\right), \\
(1, \alpha)\left(m z+r_{0}\right) a_{1}(t) & \text { otherwise, }\end{cases}
\end{aligned}
$$

where

$$
\begin{gathered}
g(z)=\left\{11-\left(\frac{94}{3 L_{0}}\right)(z-d)+\left(\frac{32}{L_{0}^{2}}\right)(z-d)^{2}-\left(\frac{32}{3 L_{0}^{3}}\right)(z-d)^{3}\right\}, \\
a_{1}(t)=1-b(\cos t-1) e^{-b t} .
\end{gathered}
$$

Using the above nondimensional variables in (2.3) and (2.5)-(2.7), we obtain

$$
\begin{gathered}
\alpha_{H}^{2} \frac{\partial u_{H}}{\partial t}=4(1+e \cos t)+4 B \cos (\omega t+\phi)-\frac{2}{r} \frac{\partial}{\partial r}\left(r \tau_{H}\right) \quad \text { if } 0 \leq r \leq R_{1}(z), \\
\alpha_{N}^{2} \frac{\partial u_{N}}{\partial t}=4(1+e \cos t)+4 B \cos (\omega t+\phi)-\frac{2}{r} \frac{\partial}{\partial r}\left(r \tau_{N}\right) \quad \text { if } R_{1}(z) \leq r \leq R(z), \\
\tau_{H}=\sqrt[r]{-\frac{1}{2} \frac{\partial u_{H}}{\partial r}}+\theta \quad \text { if } \tau_{H} \geq \theta, R_{p} \leq r \leq R_{1}(z) \\
\frac{\partial u_{H}}{\partial r}=0 \quad \text { if } \tau_{H} \leq \theta, 0 \leq r \leq R_{p} \\
\tau_{N}=-\frac{1}{2} \frac{\partial u_{N}}{\partial r} \quad \text { if } R_{1}(z) \leq r \leq R(z) .
\end{gathered}
$$

The boundary conditions (in dimensionless form) are

$$
\begin{gathered}
\tau_{H} \text { is finite at } r=0, \\
\frac{\partial u_{H}}{\partial r}=0 \quad \text { at } r=0, \\
\tau_{H}=\tau_{N} \quad \text { at } r=R_{1}(z),
\end{gathered}
$$




$$
\begin{array}{cc}
u_{H}=u_{N} \quad \text { at } r=R_{1}(z), \\
u_{N}=0 & \text { at } r=R(z) .
\end{array}
$$

The volumetric flow rate $Q$ (in nondimensional) is given by

$$
Q=4 \int_{0}^{R(z)} u(r, z, t) r d r
$$

where $Q=\bar{Q} /\left[\pi \bar{R}_{0}^{4} A_{0} / 8 \bar{\mu}_{0}\right]$ and $\bar{Q}$ is the volume flow rate.

\subsubsection{Perturbation Method of Solution}

As (2.14)-(2.18) form a system of nonlinear partial differential equations, it is not possible to obtain the exact solution to it. Perturbation method is applied to solve this system of differential equations with the boundary conditions (2.19). Since, the present study deals with the slow flow of blood (low Reynolds number flow) where the effect of pulsatile Reynolds numbers $\alpha_{H}$ and $\alpha_{N}$ are negligibly small and also they occur naturally in the nondimensional form of the momentum equation, it is more appropriate to expand the unknowns $u_{H}, u_{N}, \tau_{H}$, and $\tau_{N}$ in (2.14) and (2.18) in the perturbation series about $\alpha_{H}^{2}$ and $\alpha_{N}^{2}$. The plug core velocity $u_{p}$ and the velocity in the core region $u_{H}$ are expanded in the perturbation series of powers of $\alpha_{H}^{2}$ (where $\alpha_{H}^{2}<<1$ ) as follows:

$$
\begin{gathered}
u_{P}(z, t)=u_{0 P}(z, t)+\alpha_{H}^{2} u_{1 P}(z, t)+\cdots, \\
u_{H}(r, z, t)=u_{0 H}(r, z, t)+\alpha_{H}^{2} u_{1 H}(r, z, t)+\cdots .
\end{gathered}
$$

Similarly, we can expand $\tau_{P}, \tau_{H}$ and $R_{P}$ in powers of $\alpha_{H}^{2}$ and $u_{n}$ and $\tau_{n}$ in powers of $\alpha_{N}^{2}$. Applying the perturbation series expansions of $u_{H}$ and $\tau_{H}$ in (2.14) and then equating the constant terms and $\alpha_{H}^{2}$ terms, we obtain

$$
\begin{gathered}
\frac{\partial}{\partial r}\left(r \tau_{0 H}\right)=2[(1+e \sin t)+B \cos (\omega t+\phi)] r, \\
\frac{\partial u_{0 H}}{\partial t}=-\frac{2}{r} \frac{\partial}{\partial r}\left(r \tau_{1 H}\right) .
\end{gathered}
$$

Approximating (2.16) using binomial series and then applying the perturbation series expansions of $u_{H}$ and $\tau_{H}$ in (2.16) and thereafter equating the constant terms and $\alpha_{H}^{2}$ terms, one can get

$$
\begin{gathered}
-\frac{\partial u_{0 H}}{\partial r}=2 \tau_{0 H}^{n-1}\left[\tau_{0 H}-n \theta\right], \\
-\frac{\partial u_{1 H}}{\partial r}=2 n \tau_{0 H}{ }^{n-2} \tau_{1 H}\left[\tau_{0 H}-(n-1) \theta\right] .
\end{gathered}
$$


Substituting the perturbation series expansions of $u_{N}$ and $\tau_{N}$ in (2.15) and then equating the constant terms and $\alpha_{N}^{2}$ terms, one can obtain

$$
\begin{gathered}
\frac{\partial}{\partial r}\left(r \tau_{0 N}\right)=2[(1+e \sin t)+B \cos (\omega t+\phi)] r, \\
\frac{\partial u_{0 N}}{\partial t}=-\frac{2}{r} \frac{\partial}{\partial r}\left(r \tau_{1 N}\right) .
\end{gathered}
$$

On applying the perturbation series expansions of $u_{N}$ and $\tau_{N}$ in (2.18) and then equating the constant terms and $\alpha_{N}^{2}$ terms, we can easily get

$$
\begin{gathered}
-\frac{\partial u_{0 N}}{\partial r}=2 \tau_{0 N}, \\
-\frac{\partial u_{1 N}}{\partial r}=2 \tau_{1 N} .
\end{gathered}
$$

Use of the perturbation series expansion of $u_{H}, \tau_{H}, u_{N}$, and $\tau_{N}$ in (2.19) and then equating the constant terms and $\alpha_{H}^{2}$ and $\alpha_{N}^{2}$ terms, the boundary conditions decomposes respectively to

$$
\begin{gathered}
\tau_{0 P} \text { and } \tau_{1 P} \text { are finite at } r=0, \\
\frac{\partial u_{0 P}}{\partial r}=0, \quad \frac{\partial u_{1 P}}{\partial r}=0 \quad \text { at } r=0, \\
\tau_{0 H}=\tau_{0 N}, \quad \tau_{1 H}=\tau_{1 N} \quad \text { at } r=R_{1}(z), \\
u_{0 H}=u_{0 N}, \quad u_{1 H}=u_{1 N} \quad \text { at } r=R_{1}(z), \\
u_{0 N}=0, \quad u_{1 N}=0 \quad \text { at } r=R(z) .
\end{gathered}
$$

On solving the system of differential equations (2.22)-(2.25) with the help of boundary conditions (2.26)-(45), one can get the following expressions for the unknowns 
$\tau_{0 P}, \tau_{1 P}, u_{0 P}, u_{1 P}, u_{0 H}, u_{1 H}, \tau_{0 H}, \tau_{1 H}, u_{0 N}, u_{1 N}, \tau_{0 N}$, and $\tau_{1 N}$ (detail of obtaining these expressions is given in Sankar [33]):

$$
\begin{aligned}
& \tau_{0 P}=g(t) R_{0 P}, \\
& \tau_{0 H}=g(t) r, \\
& \tau_{0 N}=g(t) r, \\
& u_{0 N}=g(t) R^{2}\left[1-\left(\frac{r}{R}\right)^{2}\right] \\
& u_{0 H}=[g(t) R] R\left\{1-\left(\frac{R_{1}}{R}\right)^{2}\right\}+2\left[g(t) R_{1}\right]^{n} R_{1}\left[\frac{1}{(n+1)}\left\{1-\left(\frac{r}{R_{1}}\right)^{n+1}\right\}-\frac{q^{2}}{R_{1}}\left\{1-\left(\frac{r}{R_{1}}\right)^{n}\right\}\right] \text {, } \\
& u_{0 P}=[g(t) R] R\left\{1-\left(\frac{R_{1}}{R}\right)^{2}\right\}+2\left[g(t) R_{1}\right]^{n} R_{1}\left[\frac{1}{(n+1)}\left\{1-\left(\frac{R_{0 p}}{R_{1}}\right)^{n+1}\right\}-\frac{q^{2}}{R_{1}}\left\{1-\left(\frac{R_{0 p}}{R_{1}}\right)^{n}\right\}\right] \text {, } \\
& \tau_{1 P}=-\frac{1}{4}[g(t) R] D R^{2}\left(\frac{q^{2}}{R}\right)\left\{1-\left(\frac{R_{1}}{R}\right)^{2}\right\}-\left[g(t) R_{1}\right]^{n} D R_{1}^{2} \\
& \times\left[\frac{n}{2(n+1)}\left(\frac{q^{2}}{R_{1}}\right)-\frac{(n-1)}{2}\left(\frac{q^{2}}{R_{1}}\right)^{2}-\frac{n}{2(n+1)}\left(\frac{q^{2}}{R_{1}}\right)^{n+2}\right], \\
& \tau_{1 H}=-\frac{1}{4}[g(t) R] D R^{2}\left(\frac{r}{R}\right)\left\{1-\left(\frac{R_{1}}{R}\right)^{2}\right\}-\left[g(t) R_{1}\right]^{n} D R_{1}^{2} \\
& \times\left[\frac{n}{(n+1)(n+3)}\left\{\left(\frac{n+3}{2}\right)\left(\frac{r}{R_{1}}\right)-\left(\frac{r}{R_{1}}\right)^{n+2}\right\}-\frac{(n-1)}{(n+2)}\left(\frac{q^{2}}{R_{1}}\right)\right. \\
& \left.\times\left\{\left(\frac{n+2}{2}\right)\left(\frac{r}{R_{1}}\right)-\left(\frac{r}{R_{1}}\right)^{n+1}\right\}-\frac{3\left(n^{2}+2 n-2\right)}{2(n+2)(n+3)}\left(\frac{q^{2}}{R_{1}}\right)^{n+3}\left(\frac{R_{1}}{r}\right)\right], \\
& \tau_{1 N}=-[g(t) R] D R R_{1}\left[\frac{1}{4}\left(\frac{r}{R_{1}}\right)-\frac{1}{8}\left(\frac{R_{1}}{R}\right)^{2}\left(\frac{R_{1}}{r}\right)-\frac{1}{8}\left(\frac{R_{1}}{R}\right)^{2}\left(\frac{r}{R_{1}}\right)^{3}\right]-\left[g(t) R_{1}\right]^{n} D R_{1}^{2} \\
& \times\left[\frac{n}{2(n+3)}\left(\frac{R_{1}}{r}\right)-\frac{n(n-1)}{2(n+2)}\left(\frac{q^{2}}{R_{1}}\right)\left(\frac{R_{1}}{r}\right)-\frac{3\left(n^{2}+2 n-2\right)}{2(n+2)(n+3)}\left(\frac{q^{2}}{R_{1}}\right)^{n+3}\left(\frac{R_{1}}{r}\right)\right], \\
& u_{1 N}=-2[g(t) R] D R^{2} R_{1}\left[\frac{1}{8}\left(\frac{R}{R_{1}}\right)\left\{1-\left(\frac{r}{R}\right)^{2}\right\}-\frac{1}{8}\left(\frac{R_{1}}{R}\right)^{3} \log \left(\frac{R}{r}\right)-\frac{1}{32}\left(\frac{R}{R_{1}}\right)\left\{1-\left(\frac{r}{R}\right)^{4}\right\}\right] \\
& -2\left[g(t) R_{1}\right]^{n} D R_{1}^{3} \log \left(\frac{R}{r}\right)\left[\frac{n}{2(n+3)}-\frac{n(n-1)}{2(n+2)}\left(\frac{q^{2}}{R_{1}}\right)-\frac{3\left(n^{2}+2 n-2\right)}{2(n+2)(n+3)}\left(\frac{q^{2}}{R_{1}}\right)^{n+3}\right], \\
& u_{1 H}=-2[g(t) R] D R^{2} R_{1}\left[\frac{3}{32}\left(\frac{R}{R_{1}}\right)-\frac{1}{8}\left(\frac{R_{1}}{R}\right)+\frac{1}{32}\left(\frac{R_{1}}{R}\right)^{3}+\frac{1}{8}\left(\frac{R_{1}}{R}\right)^{3} \log \left(\frac{R_{1}}{R}\right)\right]
\end{aligned}
$$




$$
\begin{aligned}
& +2\left[g(t) R_{1}\right]^{n} D R_{1}^{3} \log \left(\frac{R_{1}}{R}\right)\left[\frac{n}{2(n+3)}-\frac{n(n-1)}{2(n+2)}\left(\frac{q^{2}}{R_{1}}\right)-\frac{3\left(n^{2}+2 n-2\right)}{2(n+2)(n+3)}\left(\frac{q^{2}}{R_{1}}\right)^{n+3}\right] \\
& -n\left[g(t) R_{1}\right]^{n} D R_{1} R^{2}\left\{1-\left(\frac{R_{1}}{R}\right)^{2}\right\}\left[\frac{1}{2(n+1)}\left\{1-\left(\frac{r}{R_{1}}\right)^{n+1}\right\}-\frac{(n-1)}{2 n}\left(\frac{q^{2}}{R_{1}}\right)\right. \\
& \left.\times\left\{1-\left(\frac{r}{R_{1}}\right)^{n}\right\}\right] \\
& -2 n\left[g(t) R_{1}\right]^{2 n-1} D R_{1}^{3}\left[\frac{n}{2(n+1)^{2}}\left\{1-\left(\frac{r}{R_{1}}\right)^{n+1}\right\}-\frac{(n-1)}{2(n+1)}\left(\frac{q^{2}}{R_{1}}\right)\left\{1-\left(\frac{r}{R_{1}}\right)^{n}\right\}\right. \\
& -\frac{n}{2(n+1)^{2}(n+3)}\left\{1-\left(\frac{r}{R_{1}}\right)^{2 n+2}\right\} \\
& +\frac{(n-1)\left(2 n^{2}+6 n+3\right)}{(n+1)(n+2)(n+3)(2 n+1)}\left(\frac{q^{2}}{R_{1}}\right)\left\{1-\left(\frac{r}{R_{1}}\right)^{2 n+1}\right\} \\
& -\frac{(n-1)}{2(n+1)}\left(\frac{q^{2}}{R_{1}}\right)\left\{1-\left(\frac{r}{R_{1}}\right)^{n+1}\right\}+\frac{(n-1)^{2}}{2 n}\left(\frac{q^{2}}{R_{1}}\right)^{2} \\
& \times\left\{1-\left(\frac{r}{R_{1}}\right)^{n}\right\}-\frac{(n-1)^{2}}{2 n(n+2)}\left(\frac{q^{2}}{R_{1}}\right)^{2}\left\{1-\left(\frac{r}{R_{1}}\right)^{2 n}\right\} \\
& -\frac{3\left(n^{2}+2 n-2\right)}{2(n-1)(n+2)(n+3)}\left(\frac{q^{2}}{R_{1}}\right)^{n+3}\left\{1-\left(\frac{r}{R_{1}}\right)^{n-1}\right\} \\
& \left.+\frac{3(n-1)\left(n^{2}+2 n-2\right)}{2(n-2)(n+2)(n+3)}\left(\frac{q^{2}}{R_{1}}\right)^{n+4}\left\{1-\left(\frac{r}{R_{1}}\right)^{n-2}\right\}\right], \\
& u_{1 P}=-2[g(t) R] D R^{2} R_{1}\left[\frac{3}{32}\left(\frac{R}{R_{1}}\right)-\frac{1}{8}\left(\frac{R_{1}}{R}\right)+\frac{1}{32}\left(\frac{R_{1}}{R}\right)^{3}+\frac{1}{8}\left(\frac{R_{1}}{R}\right)^{3} \log \left(\frac{R_{1}}{R}\right)\right] \\
& +2\left[g(t) R_{1}\right]^{n} D R_{1}^{3} \log \left(\frac{R_{1}}{R}\right)\left[\frac{n}{2(n+3)}-\frac{n(n-1)}{2(n+2)}\left(\frac{q^{2}}{R_{1}}\right)-\frac{3\left(n^{2}+2 n-2\right)}{2(n+2)(n+3)}\left(\frac{q^{2}}{R_{1}}\right)^{n+3}\right] \\
& -n\left[g(t) R_{1}\right]^{n} D R_{1} R^{2}\left\{1-\left(\frac{R_{1}}{R}\right)^{2}\right\}\left[\frac{1}{2(n+1)}\left\{1-\left(\frac{q^{2}}{R_{1}}\right)^{n+1}\right\}\right. \\
& \left.-\frac{(n-1)}{2 n}\left(\frac{k^{2}}{R_{1}}\right)\left\{1-\left(\frac{q^{2}}{R_{1}}\right)^{n}\right\}\right]
\end{aligned}
$$




$$
\begin{aligned}
-2 n\left[g(t) R_{1}\right]^{2 n-1} D R_{1}^{3}\left[\frac{n}{2(n+1)^{2}}\left\{1-\left(\frac{q^{2}}{R_{1}}\right)^{n+1}\right\}-\frac{(n-1)}{2(n+1)}\left(\frac{q^{2}}{R_{1}}\right)\left\{1-\left(\frac{q^{2}}{R_{1}}\right)^{n}\right\}\right. \\
+\frac{(n-1)\left(2 n^{2}+6 n+3\right)}{(n+1)(n+2)(n+3)(2 n+1)}\left(\frac{q^{2}}{R_{1}}\right)\left\{1-\left(\frac{q^{2}}{R_{1}}\right)^{2 n+1}\right\} \\
-\frac{(n-1)}{2(n+1)}\left(\frac{q^{2}}{R_{1}}\right)\left\{1-\left(\frac{q^{2}}{R_{1}}\right)^{n+1}\right\}+\frac{(n-1)^{2}}{2 n}\left(\frac{q^{2}}{R_{1}}\right)^{2} \\
\times \\
\times\left\{1-\left(\frac{q^{2}}{R_{1}}\right)^{n}\right\}-\frac{(n-1)^{2}}{2 n(n+2)}\left(\frac{q^{2}}{R_{1}}\right)^{2}\left\{1-\left(\frac{q^{2}}{R_{1}}\right)^{2 n}\right\} \\
-\frac{3\left(n^{2}+2 n-2\right)}{2(n-1)(n+2)(n+3)}\left(\frac{q^{2}}{R_{1}}\right)^{n+3}\left\{1-\left(\frac{q^{2}}{R_{1}}\right)^{n-1}\right\} \\
\left.+\frac{3(n-1)\left(n^{2}+2 n-2\right)}{2(n-2)(n+2)(n+3)}\left(\frac{q^{2}}{R_{1}}\right)^{n+4}\left\{1-\left(\frac{q^{2}}{R_{1}}\right)^{n-2}\right\}\right]
\end{aligned}
$$

where $g(t)=(1+e \cos t)+B \cos (\omega t+\phi), D=[1 / g(t)](d g(t) / d t)$, and $q^{2}=\theta / g(t)$. The expression for wall shear stress $\tau_{w}$ is obtained as follows (see [33] for detail):

$$
\begin{aligned}
\tau_{w}=[g(t) R] & +\alpha_{N}^{2}\left\{-\frac{1}{8}[g(t) R] D R^{2}\left[1-\left(\frac{R_{1}}{R}\right)^{4}\right]\right\} \\
+\alpha_{N}^{2}\{- & \frac{\left[g(t) R_{1}\right]^{n}}{2(n+2)(n+3)} D R_{1}^{2}\left(\frac{R_{1}}{R}\right) \\
& \left.\times\left[n(n+2)-n(n-1)(n+3)\left(\frac{q^{2}}{R_{1}}\right)-3\left(n^{2}+2 n-2\right)\left(\frac{q^{2}}{R_{1}}\right)^{n+3}\right]\right\} .
\end{aligned}
$$

The expression for the volume flow rate is obtained as follows (for detail see [33]):

$$
\begin{aligned}
Q= & 4[g(t) R] R^{3}\left\{1-\left(\frac{R_{1}}{R}\right)^{2}\right\}\left[\left(\frac{q^{2}}{R_{1}}\right)^{2}+\frac{1}{4}\left\{1-\left(\frac{R_{1}}{R}\right)^{2}\right\}\right] \\
& +\frac{4[g(t) R] R_{1}^{3}}{(n+2)(n+3)}\left[(n+2)-n(n+3)\left(\frac{q^{2}}{R_{1}}\right)+\left(n^{2}+2 n-2\right)\left(\frac{q^{2}}{R_{1}}\right)^{n+3}\right]
\end{aligned}
$$


Abstract and Applied Analysis

$$
\begin{aligned}
& +4 \alpha_{H}^{2}\left[-[g(t) R] D R^{2} R_{1}^{3}\left\{\frac{3}{32}\left(\frac{R}{R_{1}}\right)-\frac{1}{8}\left(\frac{R_{1}}{R}\right)+\frac{1}{32}\left(\frac{R_{1}}{R}\right)^{3}+\frac{1}{8}\left(\frac{R_{1}}{R}\right)^{3} \log \left(\frac{R_{1}}{R}\right)\right\}\right. \\
& +\left[g(t) R_{1}\right]^{n} D R_{1}^{5} \log \left(\frac{R_{1}}{R}\right)\left\{\frac{n}{2(n+3)}-\frac{n(n-1)}{2(n+2)}\left(\frac{q^{2}}{R_{1}}\right)-\frac{3\left(n^{2}+2 n-2\right)}{2(n+2)(n+3)}\left(\frac{q^{2}}{R_{1}}\right)^{n+3}\right\} \\
& -n\left[g(t) R_{1}\right]^{n} D R^{2} R_{1}^{3}\left\{1-\left(\frac{R_{1}}{R}\right)^{2}\right\}\left\{\frac{1}{4(n+3)}-\frac{(n-1)}{4(n+2)}\left(\frac{q^{2}}{R_{1}}\right)\right. \\
& \left.+\frac{\left(n^{2}+n-5\right)}{4(n+2)(n+3)}\left(\frac{q^{2}}{R_{1}}\right)^{n+3}\right\} \\
& -n\left[g(t) R_{1}\right]^{2 n-1} D R_{1}^{5}\left\{\frac{n}{2(n+2)(n+3)}-\frac{n(n-1)\left(4 n^{2}+12 n+5\right)}{(n+2)(n+3)(2 n+1)(2 n+3)}\left(\frac{q^{2}}{R_{1}}\right)\right. \\
& +\frac{n(n-1)^{2}}{2(n+1)(n+2)}\left(\frac{q^{2}}{R_{1}}\right)^{2}+\frac{\left(n^{3}-2 n^{2}-11 n+6\right)}{2(n+1)(n+2)(n+3)}\left(\frac{q^{2}}{R_{1}}\right)^{n+3} \\
& -\frac{(n-1)\left(n^{3}-2 n^{2}-11 n+6\right)}{2 n(n+2)(n+3)}\left(\frac{q^{2}}{R_{1}}\right)^{n+4} \\
& \left.\left.-\frac{\left(4 n^{5}+14 n^{4}-8 n^{3}-45 n^{2}-3 n+18\right)}{2 n(n+1)(n+2)(n+3)(2 n+3)}\left(\frac{q^{2}}{R_{1}}\right)^{2 n+4}\right\}\right] \\
& +4 \alpha_{N}^{2}\left[-[g(t) R] D R^{4} R_{1}\left\{\frac{1}{24}\left(\frac{R}{R_{1}}\right)-\frac{3}{32}\left(\frac{R_{1}}{R}\right)+\frac{5}{96}\left(\frac{R_{1}}{R}\right)^{5}\right.\right. \\
& \left.-\frac{1}{8}\left(\frac{R_{1}}{R}\right)^{3}\left(\log R_{1}\right)\left\{1-\left(\frac{R_{1}}{R}\right)^{2}\right\}\right\} \\
& \text { - }\left[g(t) R_{1}\right]^{n} D R^{2} R_{1}^{3}\left\{1-\left(\frac{R_{1}}{R}\right)^{2}\right\}\left(1+2 \log R_{1}\right) \\
& \left.\times\left\{\frac{n}{4(n+3)}-\frac{n(n-1)}{4(n+2)}\left(\frac{q^{2}}{R_{1}}\right)-\frac{3\left(n^{2}+2 n-2\right)}{4(n+2)(n+3)}\left(\frac{q^{2}}{R_{1}}\right)^{n+3}\right\}\right] .
\end{aligned}
$$

The expression for plug core radius is obtained as follows (detail of obtaining this expression is given in [33]):

$$
\begin{aligned}
R_{P}= & k^{2}+\left(\frac{D \alpha_{H}^{2} R^{2}}{4}\right)[g(t) R]\left(\frac{q^{2}}{R}\right)\left\{1-\left(\frac{R_{1}}{R}\right)^{2}\right\} \\
& +\frac{n D \alpha_{H}^{2} R_{1}^{2}}{2(n+1)}\left[g(t) R_{1}\right]^{n}\left\{\left(\frac{q^{2}}{R_{1}}\right)-\frac{\left(n^{2}-1\right)}{n}\left(\frac{q^{2}}{R_{1}}\right)^{2}-\left(\frac{q^{2}}{R_{1}}\right)^{n+2}\right\} .
\end{aligned}
$$


The resistance to flow in the artery is given by

$$
\Lambda=\frac{[1+e \cos t]}{Q}
$$

when $R_{1}=R$; the present model reduces to the single-fluid H-B model and in this case, the expressions obtained in the present model for velocity, shear stress, wall shear stress, flow rate, and plug core radius are in good agreement with those of Sankar and Ismail [14].

\subsection{Two-Fluid Casson Fluid Model}

\subsubsection{Governing Equations and Boundary Conditions}

Equations (2.1)-(2.2) which mathematically define the geometry of the tapered artery with overlapping stenosis are assumed in this subsection. The momentum equations governing the flow in the core region and peripheral layer region simplify to [34]

$$
\begin{gathered}
\bar{\rho}_{C} \frac{\partial \bar{u}_{C}}{\partial \bar{t}}=-\frac{\partial \bar{p}}{\partial \bar{z}}-\frac{1}{\bar{r}} \frac{\partial}{\partial \bar{r}}\left(\overline{r \tau}_{C}\right)+\bar{F}(\bar{t}) \quad \text { in } 0 \leq \bar{r} \leq \bar{R}_{1}(\bar{z}), \\
\bar{\rho}_{N} \frac{\partial \bar{u}_{N}}{\partial \bar{t}}=-\frac{\partial \bar{p}}{\partial \bar{z}}-\frac{1}{\bar{r}} \frac{\partial}{\partial \bar{r}}\left(\bar{r}_{N}\right)+\bar{F}(\bar{t}) \quad \text { in } \bar{R}_{1}(\bar{z}) \leq \bar{r} \leq \bar{R}(\bar{z}), \\
0=-\frac{\partial \bar{p}}{\partial \bar{r}},
\end{gathered}
$$

where the shear stress $\bar{\tau}=\left|\bar{\tau}_{\overline{r z}}\right|=-\bar{\tau}_{\overline{r z}}$ (since $\bar{\tau}=\bar{\tau}_{C}$ or $\bar{\tau}=\bar{\tau}_{N}$ ); $\bar{\tau}_{C}$ and $\bar{\tau}_{N}$ are the shear stress of the fluid in the core region (Casson fluid) and peripheral layer region (Newtonian fluid), respectively; $\bar{u}_{C}$ and $\bar{u}_{N}$ are the axial velocity of the fluid in the core region and peripheral layer region, respectively; $\bar{\rho}_{C}$ and $\bar{\rho}_{N}$ are the densities of the Casson fluid and Newtonian fluid, respectively; $\bar{p}$ is the pressure; $\bar{t}$ is the time. Equations (2.9) and (2.10) which define mathematically the body acceleration term $\bar{F}(\bar{t})$ and pressure gradient $-(\partial \bar{p} / \partial \bar{z})$ are assumed in this subsection. The constitutive equations of the fluids in motion in the core region (Casson fluid) and peripheral layer region (Newtonian fluid) are

$$
\begin{gathered}
\sqrt{\bar{\tau}_{C}}=\sqrt{-\bar{\mu}_{C} \frac{\partial \bar{u}_{C}}{\partial \bar{r}}+\sqrt{\bar{\tau}_{y}} \quad \text { if } \bar{\tau}_{C} \geq \bar{\tau}_{y}, \bar{R}_{p} \leq \bar{r} \leq \bar{R}_{1}(\bar{z}),} \\
\frac{\partial \bar{u}_{C}}{\partial \bar{r}}=0 \quad \text { if } \bar{\tau}_{C} \leq \bar{\tau}_{y}, 0 \leq \bar{r} \leq \bar{R}_{p} \\
\bar{\tau}_{N}=-\bar{\mu}_{N}\left(\frac{\partial \bar{u}_{N}}{\partial \bar{r}}\right) \quad \text { if } \bar{R}_{1}(\bar{z}) \leq \bar{r} \leq \bar{R}(\bar{z})
\end{gathered}
$$


where $\bar{\tau}_{y}$ is the yield stress; $\bar{R}_{P}$ is the plug core radius; $\bar{\mu}_{C}$ and $\bar{\mu}_{N}$ are the viscosities of the Casson fluid and Newtonian fluid, respectively. The appropriate boundary conditions of the two-fluid flow are

$$
\begin{gathered}
\bar{\tau}_{C} \text { is finite, } \frac{\partial \bar{u}_{C}}{\partial \bar{r}}=0 \text { at } \bar{r}=0, \\
\bar{u}_{N}=0 \quad \text { at } r=\bar{R}, \\
\bar{\tau}_{C}=\bar{\tau}_{N}, \quad \bar{u}_{C}=\bar{u}_{N} \quad \text { at } \bar{r}=\bar{R}_{1} .
\end{gathered}
$$

\subsubsection{Nondimensionalization}

Let us introduce the following nondimensional variables:

$$
\begin{gathered}
z=\frac{\bar{z}}{\bar{R}_{0}}, \quad R(z)=\frac{\bar{R}(\bar{z})}{\bar{R}_{0}}, \quad R_{1}(z)=\frac{\bar{R}_{1}(\bar{z})}{\bar{R}_{0}}, \quad r=\frac{\bar{r}}{\bar{R}_{0}}, \quad u_{C}=\frac{\bar{u}_{C}}{A_{0} \bar{R}_{0}^{2} / 4 \bar{\mu}_{C}}, \\
u_{N}=\frac{\bar{u}_{N}}{A_{0} \bar{R}_{0}^{2} / 4 \bar{\mu}_{N}}, \quad \tau_{C}=\frac{\bar{\tau}_{C}}{A_{0} \bar{R}_{0} / 2}, \quad \alpha_{C}^{2}=\frac{\bar{R}_{0}^{2} \bar{\omega}_{p} \bar{\rho}_{C}}{\bar{\mu}_{C}}, \quad \tau_{N}=\frac{\bar{\tau}_{N}}{A_{0} \bar{R}_{0} / 2}, \\
\theta=\frac{\bar{\tau}_{y}}{A_{0} \bar{R}_{0} / 2}, \quad \alpha_{N}^{2}=\frac{\bar{R}_{0}^{2} \bar{\omega}_{p} \bar{\rho}_{N}}{\bar{\mu}_{N}}, \quad R_{p}=\frac{\bar{R}_{p}}{\bar{R}_{0}}, \quad \delta_{p}=\frac{\bar{\delta}_{p}}{\bar{R}_{0}}, \quad \delta_{C}=\frac{\bar{\delta}_{C}}{\bar{R}_{0}}, \\
\quad e=\frac{A_{1}}{A_{0}}, \quad B=\frac{a_{0}}{A_{0}}, \quad \omega=\frac{\bar{\omega}_{b}}{\bar{\omega}_{p}}, \quad t=\bar{\omega}_{p} \bar{t},
\end{gathered}
$$

where $\alpha_{C}$ and $\alpha_{N}$ are the pulsatile Reynolds numbers of the Casson fluid and Newtonian fluid, respectively. Using the nondimensional variables in the momentum equations (2.32) and (2.33) and the constitutive equations (2.35), the simplified form of these equations can be obtained respectively as follows:

$$
\begin{gathered}
\alpha_{C}^{2} \frac{\partial u_{C}}{\partial t}=4(1+e \cos t)+4 B \cos (\omega t+\phi)-\frac{2}{r} \frac{\partial}{\partial r}\left(r \tau_{C}\right) \quad \text { if } 0 \leq r \leq R_{1}(z) \\
\alpha_{N}^{2} \frac{\partial u_{N}}{\partial t}=4(1+e \cos t)+4 B \cos (\omega t+\phi)-\frac{2}{r} \frac{\partial}{\partial r}\left(r \tau_{N}\right) \quad \text { if } R_{1}(z) \leq r \leq R(z) \\
\sqrt{\tau_{C}}=\sqrt{-\frac{1}{2} \frac{\partial u_{C}}{\partial r}}+\sqrt{\theta} \quad \text { if } \tau_{C} \geq \theta, R_{p} \leq r \leq R_{1}(z) \\
\frac{\partial u_{C}}{\partial r}=0 \quad \text { if } \quad \tau_{C} \leq \theta, 0 \leq r \leq R_{p} \\
\tau_{N}=-\frac{1}{2} \frac{\partial u_{N}}{\partial r} \quad \text { if } R_{1}(z) \leq r \leq R(z) .
\end{gathered}
$$


Using the nondimensional variables, the boundary conditions become

$$
\begin{gathered}
\tau_{C} \text { is finite, } \frac{\partial u_{C}}{\partial r}=0 \quad \text { at } r=0, \\
\tau_{C}=\tau_{N}, \quad u_{C}=u_{N} \quad \text { at } r=R, \\
u_{N}=0 \quad \text { at } r=R .
\end{gathered}
$$

Equations (2.12)-(2.13) which mathematically defines the nondimensional form of the geometry of the segment of the tapered artery with overlapping stenosis is assumed in this subsection.

The nondimensional volume flow rate $Q$ is given by

$$
Q=4 \int_{0}^{R(z)} u(r, z, t) r d r
$$

where $Q=\bar{Q} /\left[\pi \bar{R}_{0}^{4} A_{0} / 8 \bar{\mu}_{C}\right] ; \bar{Q}$ is the volume flow rate.

\subsubsection{Perturbation Method of Solution}

As it is not possible to find an exact solution to the system of nonlinear partial differential equations (2.38)-(2.42), perturbation method is applied to obtain the asymptotic solution to the unknowns $u_{C}, u_{N}, \tau_{C}$, and $\tau_{N}$. Since, the present study deals with the slow flow of blood (low Reynolds number flow) where the effect of pulsatile Reynolds numbers $\alpha_{C}$ and $\alpha_{N}$ are negligibly small and also they occur naturally in the nondimensional form of the momentum equation, it is appropriate to expand (2.38)-(2.42) in the perturbation series about $\alpha_{C}^{2}$ and $\alpha_{N}^{2}$. The plug core velocity $u_{p}$ and the velocity in the core region $u_{C}$ are expanded in the perturbation series of $\alpha_{C}^{2}$ as follows (where $\alpha_{C}^{2}<<1$ ):

$$
\begin{gathered}
u_{p}(z, t)=u_{0 p}(z, t)+\alpha_{C}^{2} u_{1 p}(z, t)+\cdots, \\
u_{C}(r, z, t)=u_{0 C}(r, z, t)+\alpha_{C}^{2} u_{1 C}(r, z, t)+\cdots .
\end{gathered}
$$

Similarly, one may expand $u_{N}, \tau_{P}, \tau_{C}, \tau_{N}$, and the plug core radius $R_{P}$ in the perturbation series about $\alpha_{C}^{2}$ and $\alpha_{N}^{2}$, where $\alpha_{N}^{2}<1$. Using the perturbation series expansions of $u_{C}$ and $\tau_{C}$ in (2.38) and then equating the constant terms and $\alpha_{C}^{2}$ terms, the momentum equation of the core region decomposes to

$$
\begin{gathered}
\frac{\partial}{\partial r}\left(r \tau_{0 C}\right)=2[1+e \sin t+B \cos (\omega t+\phi)] r, \\
\frac{\partial u_{0 C}}{\partial t}=-\frac{2}{r} \frac{\partial}{\partial r}\left(r \tau_{1 C}\right) .
\end{gathered}
$$


Applying the perturbation series expansions of $u_{C}$ and $\tau_{C}$ in (2.40) and then equating the constant terms and $\alpha_{C}^{2}$ terms, the constitutive equation of the core region simplifies to

$$
\begin{gathered}
-\frac{\partial u_{0 C}}{\partial r}=2\left(\tau_{0 C}-2 \sqrt{\theta \tau_{0 C}}+\theta\right), \\
-\frac{\partial u_{1 C}}{\partial r}=2 \tau_{1 C}\left(1-\sqrt{\frac{\theta}{\tau_{0 C}}}\right) .
\end{gathered}
$$

Similarly, substituting the perturbation series expansions of $u_{N}$ and $\tau_{N}$ in (2.39) and then equating the constant terms and $\alpha_{N}^{2}$ terms, the momentum equation of the peripheral region decomposes to

$$
\begin{gathered}
\frac{\partial}{\partial r}\left(r \tau_{0 N}\right)=2[1+e \sin t+B \cos (\omega t+\phi)] r, \\
\frac{\partial u_{0 N}}{\partial t}=-\frac{2}{r} \frac{\partial}{\partial r}\left(r \tau_{1 N}\right) .
\end{gathered}
$$

Applying the perturbation series expansions of $u_{N}$ and $\tau_{N}$ in (2.42) and then equating the constant terms and $\alpha_{N}^{2}$ terms, the constitutive equation of the peripheral region reduces to

$$
\begin{aligned}
& -\frac{\partial u_{0 N}}{\partial r}=2 \tau_{0 N}, \\
& -\frac{\partial u_{1 N}}{\partial r}=2 \tau_{1 N} .
\end{aligned}
$$

Using the perturbation series expansions of $u_{C}, u_{N}, \tau_{C}$, and $\tau_{N}$ in (2.43) and then equating the constant terms and $\alpha_{C}^{2}$ and $\alpha_{N}^{2}$ terms, one can obtain

$\tau_{0 P}$ and $\tau_{1 P}$ are finite at $r=0$,

$$
\begin{gathered}
\frac{\partial u_{0 P}}{\partial r}=0, \quad \frac{\partial u_{1 P}}{\partial r}=0 \quad \text { at } r=0, \\
\tau_{0 C}=\tau_{0 N}, \quad \tau_{1 C}=\tau_{1 N} \quad \text { at } r=R_{1}(z), \\
u_{0 C}=u_{0 N}, \quad u_{1 C}=u_{1 N} \quad \text { at } r=R_{1}(z), \\
u_{0 N}=0, \quad u_{1 N}=0 \quad \text { at } r=R(z) .
\end{gathered}
$$


Solving the system of (2.46)-(2.49) using the boundary conditions (2.50), one can obtain the following expressions for the unknowns $u_{0 P}, u_{1 P}, \tau_{0 P}, \tau_{1 P}, u_{0 C}, u_{1 C}, \tau_{0 C}, \tau_{1 C}, u_{0 N}, u_{1 N}, \tau_{0 N}$, and $\tau_{1 N}$ :

$$
\begin{aligned}
& \tau_{0 p}=g(t) R_{0 p}, \\
& \tau_{0 C}=g(t) r, \\
& \tau_{0 N}=g(t) r, \\
& u_{0 N}=g(t) R^{2}\left[1-\left(\frac{r}{R}\right)^{2}\right], \\
& u_{0 C}=g(t) R^{2}\left(\left\{1-\left(\frac{R_{1}}{R}\right)^{2}\right\}+\left(\frac{R_{1}}{R}\right)^{2}\right. \\
& \times\left[1-\left(\frac{r}{R_{1}}\right)^{2}-\frac{8}{3} \sqrt{\frac{q^{2}}{R_{1}}}\left\{1-\left(\frac{r}{R_{1}}\right)^{3 / 2}\right\}+2\left(\frac{q^{2}}{R_{1}}\right)\left\{1-\left(\frac{r}{R_{1}}\right)\right\}\right], \\
& u_{0 P}=g(t) R^{2}\left(\left\{1-\left(\frac{R_{1}}{R}\right)^{2}\right\}+\left(\frac{R_{1}}{R}\right)^{2}\right. \\
& \left.\times\left[1-\left(\frac{R_{0 P}}{R_{1}}\right)^{2}-\frac{8}{3} \sqrt{\frac{q^{2}}{R_{1}}}\left\{1-\left(\frac{R_{0 P}}{R_{1}}\right)^{3 / 2}\right\}+2\left(\frac{q^{2}}{R_{1}}\right)\left\{1-\left(\frac{R_{0 P}}{R_{1}}\right)\right\}\right]\right) \\
& \tau_{1 p}=-g(t) D R^{3}\left\{\frac{1}{4}\left(\frac{q^{2}}{R}\right)\left[1-\left(\frac{R_{1}}{R}\right)^{2}\right]+\left(\frac{R_{1}}{R}\right)^{3}\left(\frac{q^{2}}{R_{1}}\right)\left[\frac{1}{4}-\frac{1}{3} \sqrt{\frac{q^{2}}{R_{1}}}+\frac{1}{12}\left(\frac{q^{2}}{R_{1}}\right)^{2}\right]\right\} \\
& \tau_{1 C}=-g(t) D R^{3}\left\{\frac{1}{4}\left(\frac{r}{R}\right)\left[1-\left(\frac{R_{1}}{R}\right)^{2}\right]-\frac{1}{8}\left(\frac{R_{1}}{R}\right)^{3}\right. \\
& \times\left[2\left(\frac{r}{R_{1}}\right)-\left(\frac{r}{R_{1}}\right)^{3}-\left(\frac{q^{2}}{R_{1}}\right)^{4}\left(\frac{r}{R_{1}}\right)^{-1}-\frac{8}{21} \sqrt{\frac{q^{2}}{R_{1}}}\right. \\
& \left.\left.\times\left(7\left(\frac{r}{R_{1}}\right)-4\left(\frac{r}{R_{1}}\right)^{5 / 2}-3\left(\frac{q^{2}}{R_{1}}\right)^{7 / 2}\left(\frac{r}{R_{1}}\right)^{-1}\right)\right]\right\}, \\
& \tau_{1 N}=-g(t) D R^{2} R_{1}\left\{\left[\frac{1}{4}\left(\frac{r}{R_{1}}\right)-\frac{1}{8}\left(\frac{R_{1}}{R}\right)^{2}\left(\frac{r}{R_{1}}\right)^{-1}-\frac{1}{8}\left(\frac{R_{1}}{R}\right)^{2}\left(\frac{r}{R_{1}}\right)^{3}\right]\right. \\
& \left.+\left(\frac{r}{R_{1}}\right)^{-1}\left(\frac{R_{1}}{R}\right)^{2}\left[\frac{1}{8}-\frac{1}{7} \sqrt{\frac{q^{2}}{R_{1}}}+\frac{1}{56}\left(\frac{q^{2}}{R_{1}}\right)^{4}\right]\right\}
\end{aligned}
$$




$$
\begin{aligned}
& u_{1 N}=-g(t) D R^{3} R_{1}\left\{\left[\frac{1}{4}\left(\frac{R_{1}}{R}\right)^{-1}\left\{1-\left(\frac{r}{R}\right)^{2}\right\}-\frac{1}{4}\left(\frac{R_{1}}{R}\right)^{3} \log \left(\frac{r}{R}\right)^{-1}-\frac{1}{16}\left(\frac{R_{1}}{R}\right)^{-1}\left\{1-\left(\frac{r}{R}\right)^{4}\right\}\right]\right. \\
& \left.-\left(\frac{R_{1}}{R}\right)^{3} \log \left(\frac{r}{R}\right)\left[\frac{1}{4}-\frac{2}{7} \sqrt{\frac{q^{2}}{R_{1}}}+\frac{1}{28}\left(\frac{q^{2}}{R_{1}}\right)^{4}\right]\right\} \\
& u_{1 C}=-g(t) D R^{3} R_{1}\left(\left[\frac{3}{16}\left(\frac{R_{1}}{R}\right)^{-1}-\frac{1}{4}\left(\frac{R_{1}}{R}\right)+\frac{1}{16}\left(\frac{R_{1}}{R}\right)^{3}+\frac{1}{4}\left(\frac{R_{1}}{R}\right)^{3} \log \left(\frac{R_{1}}{R}\right)\right]\right. \\
& -\left(\frac{R_{1}}{R}\right)^{3} \log \left(\frac{R_{1}}{R}\right)\left[\frac{1}{4}-\frac{2}{7} \sqrt{\frac{q^{2}}{R_{1}}}+\frac{1}{28}\left(\frac{q^{2}}{R_{1}}\right)^{4}\right] \\
& +\left(\frac{R_{1}}{R}\right)\left\{1-\left(\frac{R_{1}}{R}\right)^{2}\right\}\left[\frac{1}{4}\left\{1-\left(\frac{r}{R_{1}}\right)\right\}-\frac{1}{3} \sqrt{\frac{q^{2}}{R_{1}}}\left\{1-\left(\frac{r}{R_{1}}\right)^{3 / 2}\right\}\right] \\
& +\left(\frac{R_{1}}{R}\right)^{3}\left[\frac{1}{4}\left\{1-\left(\frac{r}{R_{1}}\right)^{2}\right\}-\frac{1}{3} \sqrt{\frac{q^{2}}{R_{1}}}\left\{1-\left(\frac{r}{R_{1}}\right)^{3 / 2}\right\}-\frac{1}{16}\left\{1-\left(\frac{r}{R_{1}}\right)^{4}\right\}\right. \\
& +\frac{53}{294} \sqrt{\frac{q^{2}}{R_{1}}}\left\{1-\left(\frac{r}{R_{1}}\right)^{7 / 2}\right\}-\frac{1}{3}\left\{1-\left(\frac{r}{R_{1}}\right)^{2}\right\}+\frac{4}{9}\left(\frac{q^{2}}{R_{1}}\right) \\
& \times\left\{1-\left(\frac{r}{R_{1}}\right)^{3 / 2}\right\}-\frac{8}{63}\left(\frac{q^{2}}{R_{1}}\right)\left\{1-\left(\frac{r}{R_{1}}\right)^{3}\right\} \\
& \left.\left.-\frac{1}{28}\left(\frac{q^{2}}{R_{1}}\right)^{4} \log \left(\frac{r}{R_{1}}\right)+\frac{1}{14}\left(\frac{q^{2}}{R_{1}}\right)^{9 / 2}\left\{1-\left(\frac{r}{R_{1}}\right)^{-1 / 2}\right\}\right]\right) \\
& u_{1 P}=-g(t) D R^{3} R_{1}\left(\left[\frac{3}{16}\left(\frac{R_{1}}{R}\right)^{-1}-\frac{1}{4}\left(\frac{R_{1}}{R}\right)+\frac{1}{16}\left(\frac{R_{1}}{R}\right)^{3}+\frac{1}{4}\left(\frac{R_{1}}{R}\right)^{3} \log \left(\frac{R_{1}}{R}\right)\right]\right. \\
& -\left(\frac{R_{1}}{R}\right)^{3} \log \left(\frac{R_{1}}{R}\right)\left[\frac{1}{4}-\frac{2}{7} \sqrt{\frac{q^{2}}{R_{1}}}+\frac{1}{28}\left(\frac{q^{2}}{R_{1}}\right)^{4}\right] \\
& +\left(\frac{R_{1}}{R}\right)\left\{1-\left(\frac{R_{1}}{R}\right)^{2}\right\}\left[\frac{1}{4}\left\{1-\left(\frac{q^{2}}{R_{1}}\right)\right\}-\frac{1}{3} \sqrt{\frac{q^{2}}{R_{1}}}\left\{1-\left(\frac{q^{2}}{R_{1}}\right)^{3 / 2}\right\}\right] \\
& +\left(\frac{R_{1}}{R}\right)^{3}\left[\frac{1}{4}\left\{1-\left(\frac{q^{2}}{R_{1}}\right)^{2}\right\}-\frac{1}{3} \sqrt{\frac{q^{2}}{R_{1}}}\left\{1-\left(\frac{q^{2}}{R_{1}}\right)^{3 / 2}\right\}\right. \\
& -\frac{1}{16}\left\{1-\left(\frac{q^{2}}{R_{1}}\right)^{4}\right\}+\frac{53}{294} \sqrt{\frac{q^{2}}{R_{1}}}\left\{1-\left(\frac{q^{2}}{R_{1}}\right)^{7 / 2}\right\}
\end{aligned}
$$




$$
\begin{aligned}
& -\frac{1}{3}\left\{1-\left(\frac{q^{2}}{R_{1}}\right)^{2}\right\}+\frac{4}{9} \\
& \times\left(\frac{q^{2}}{R_{1}}\right)\left\{1-\left(\frac{q^{2}}{R_{1}}\right)^{3 / 2}\right\}-\frac{8}{63}\left(\frac{q^{2}}{R_{1}}\right)\left\{1-\left(\frac{q^{2}}{R_{1}}\right)^{3}\right\} \\
& \left.\left.-\frac{1}{28}\left(\frac{q^{2}}{R_{1}}\right)^{4} \log \left(\frac{q^{2}}{R_{1}}\right)+\frac{1}{14}\left(\frac{q^{2}}{R_{1}}\right)^{9 / 2}\left\{1-\left(\frac{q^{2}}{R_{1}}\right)^{-1 / 2}\right\}\right]\right),
\end{aligned}
$$

where $g(t)=(1+e \cos t)+B \cos (\omega t+\phi), q^{2}=\left.r\right|_{\tau_{0 P}=\theta}=R_{0 p}=\theta / g(t) ; \mathrm{D}=[1 / g(t)](d g(t) / d t)$. The expression for wall shear stress $\tau_{w}$ can be obtained as follows (for detail, see [34]):

$$
\tau_{w}=g(t)\left\{R-\frac{1}{8} D R^{3} \alpha_{N}^{2}\left[1-\left(\frac{R_{1}}{R}\right)^{4}\right]-\frac{1}{8} D R_{1}^{3} \alpha_{N}^{2}\left(\frac{R_{1}}{R}\right)\left[1-\frac{8}{7} \sqrt{\frac{q^{2}}{R_{1}}}+\frac{1}{7}\left(\frac{q^{2}}{R_{1}}\right)^{4}\right]\right\}
$$

The expression for volume flow rate is obtained as follows (see [34] for detail):

$$
\begin{aligned}
Q=g(t) R^{4}\left\{\left[1-\left(\frac{R_{1}}{R}\right)^{2}\right]\right. & {\left.\left[1+3\left(\frac{R_{1}}{R}\right)^{2}\right]+\left(\frac{R_{1}}{R}\right)^{4}\left[1-\frac{16}{7} \sqrt{\frac{q^{2}}{R_{1}}}+\frac{4}{3}\left(\frac{q^{2}}{R_{1}}\right)-\frac{1}{21}\left(\frac{q^{2}}{R_{1}}\right)^{4}\right]\right\} } \\
-\alpha_{C}^{2} g(t) D R^{3} R_{1}^{3}\{[ & \left.\frac{3}{8}\left(\frac{R_{1}}{R}\right)^{-1}-\frac{1}{2}\left(\frac{R_{1}}{R}\right)+\frac{1}{8}\left(\frac{R_{1}}{R}\right)^{3}+\frac{1}{2}\left(\frac{R_{1}}{R}\right)^{3} \log \left(\frac{R_{1}}{R}\right)\right]-\left(\frac{R_{1}}{R}\right)^{3} \\
\times & \log \left(\frac{R_{1}}{R}\right)\left[\frac{1}{2}-\frac{4}{7} \sqrt{\frac{q^{2}}{R_{1}}}+\frac{1}{14}\left(\frac{q^{2}}{R_{1}}\right)^{4}\right]+\left(\frac{R_{1}}{R}\right)\left[1-\left(\frac{R_{1}}{R}\right)^{2}\right] \\
\times & {\left.\left.\left.\left[\frac{1}{4}-\frac{2}{7} \sqrt{\frac{q^{2}}{R_{1}}}+\frac{1}{28}\left(\frac{q^{2}}{R_{1}}\right)^{4}\right]+\left(\frac{R_{1}}{R}\right)^{3}\right)^{4}\right)^{4}\right] } \\
\times & {\left[\frac{1}{6}-\frac{30}{77} \sqrt{\frac{q^{2}}{R_{1}}}+\frac{8}{35}\left(\frac{q^{2}}{R_{1}}\right)^{-}-\frac{1}{3}\left(\frac{q^{2}}{R_{1}}\right)^{5 / 2}+\frac{1}{14}\left(\frac{q^{2}}{R_{1}}\right)^{4}\right] } \\
+ & \frac{5}{21}\left(\frac{q^{2}}{R_{1}}\right)^{9 / 2}-\frac{41}{770}\left(\frac{q^{2}}{R_{1}}\right)^{6}-\frac{1}{14}\left(\frac{q^{2}}{R_{1}}\right)^{6} \log \left(\frac{q^{2}}{R_{1}}\right) \\
+ & \frac{1}{14}\left(\frac{q^{2}}{R_{1}}\right)^{4}\left[1-\left(\frac{q^{2}}{R_{1}}\right)^{2}\right] \log k-\alpha_{N}^{2} g(t) D R^{5} R_{1}
\end{aligned}
$$




$$
\begin{aligned}
& \times\left\{\left[\frac{1}{6}\left(\frac{R_{1}}{R}\right)^{-1}-\frac{3}{8}\left(\frac{R_{1}}{R}\right)+\frac{5}{24}\left(\frac{R_{1}}{R}\right)^{5}-\frac{1}{2}\left(\frac{R_{1}}{R}\right)^{3}\left(1-\left(\frac{R_{1}}{R}\right)^{2}\right) \log R_{1}\right]\right. \\
& \left.\quad+\left(\frac{R_{1}}{R}\right)^{4}\left[1-\left(\frac{R_{1}}{R}\right)^{2}\right]\left(1+2 \log R_{1}\right)\left[\frac{1}{4}-\frac{2}{7} \sqrt{\frac{q^{2}}{R_{1}}}+\frac{1}{28}\left(\frac{q^{2}}{R_{1}}\right)^{4}\right]\right\} .
\end{aligned}
$$

The expression for the plug core radius $R_{P}$ can be obtained as follows (see [34] for details):

$$
R_{p}=q^{2}-\frac{1}{4} D \alpha_{C}^{2} R^{3}\left\{\left(\frac{q^{2}}{R}\right)^{2}\left[1-\left(\frac{R_{1}}{R}\right)^{2}\right]^{2}+\left(\frac{R_{1}}{R}\right)^{3}\left[\left(\frac{q^{2}}{R_{1}}\right)-\frac{4}{3}\left(\frac{q^{2}}{R_{1}}\right)^{3 / 2}+\frac{1}{3}\left(\frac{q^{2}}{R_{1}}\right)^{3}\right]\right\} .
$$

The longitudinal impedance to flow is given by

$$
\Lambda=\frac{[1+e \cos t]}{Q}
$$

When $R_{1}=R$, the present model reduces to the single-fluid Casson model and in this case, the expressions obtained in the present model for velocity, shear stress, wall shear stress, flow rate, and plug core radius are identical with those of Nagarani and Sarojamma [16].

\section{Numerical Simulation of the Results}

The objective of the present mathematical analysis is to compare the two-fluid H-B and Casson models for blood flow in narrow tapered arteries with mild overlapping stenosis and bring out the advantageous of using the two-fluid H-B fluid model rather than the twofluid Casson fluid. It is also aimed to bring out the effects of body acceleration, tapering of the artery, depth of the stenosis, yield stress, power law index, lead angle, frequency ratio, and pressure gradient on the physiologically important flow quantities such as plug core radius, plug flow velocity, velocity distribution, flow rate, wall shear stress, and longitudinal impedance to flow. Range of the values of various parameters used in this mathematical analysis is grouped below [33-36].

Angle of tapering $\psi$ : $-0.1-0.1$; amplitude parameter of the artery radius (involved in the time dependent term) $b$ : $0-0.5$; yield stress $\theta$ : $0-0.2$; power law index $n$ : 0.95-1.05; pressure gradient $e$ : $0-1$; body acceleration $B$ : $0-2$; frequency parameter $\omega$ : $0-1$; pulsatile Reynolds numbers $\alpha_{H}$ and $\alpha_{C}: 0.2$; lead angle $\phi: 0.2-0.5$; maximum depth of the stenosis in the peripheral layer region $\delta_{P}: 0-0.15$. Stenosis location in axial direction $z: 2-3.5$.

The pulsatile Reynolds number ratio $\alpha$ is defined as $\alpha=\alpha_{N} / \alpha_{H}$ or $\alpha=\alpha_{N} / \alpha_{C}$ and its value is taken as the same as those of $\alpha_{H}$ or $\alpha_{C}$ [29]. The value of $\alpha_{N}$ is computed from these relations. The value of the ratio $\beta$ of central core radius $\beta \bar{R}_{0}$ to the normal artery radius $\bar{R}_{0}$ in the unobstructed artery is taken in the range 0.925-0.975 [30]. Following Shukla et al. [37], relations $R_{1}=\beta R$ and $\delta_{c}=\beta_{\delta p}$ are used to compute $R_{1}$ and $\delta_{c}$. The time for one complete cardiac cycle is 0.85 second [36] and in our numerical simulations, the time range of $0-0.85$ 


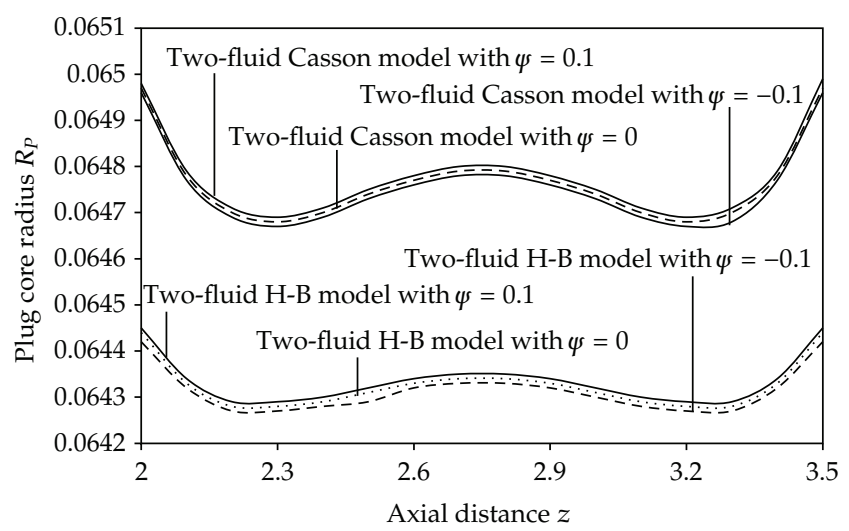

Figure 2: Variation of plug core radius with axial distance for two-fluid H-B and Casson models and different angle of tapering with $b=0.1, t=60^{\circ}, \delta_{P}=\theta=0.1, e=0.5, B=1, \omega=1, \phi=\alpha_{H}=\alpha_{C}=0.2$, and $\beta=n=0.95$.

second is converted into one time cycle in degree measure ranging $0^{\circ}-360^{\circ}$. From Figure $1(\mathrm{~b})$, it is noted that the angles of tapering $\psi=-0.1$ and $\psi=0.1$ correspond to the converging and diverging of the arterial diameter in the downstream of the flow, respectively, while $\psi=0$ corresponds to the nontapered artery.

\subsection{Plug Core Radius}

Figure 2 depicts the variation of the plug core radius with axial distance for different angles of tapering and two-fluid H-B and Casson models with $b=0.1, t=60^{\circ}, \delta_{P}=\theta=0.1, e=$ $0.5, B=1, \phi=\alpha_{H}=\alpha_{C}=0.2, \beta=n=0.95$, and $\omega=1$. It is observed that the plug core radius of the artery decreases rapidly with the increases of the axial variable $z$ from 0 to 2.3 and then it increases slowly with the increase of $z$ from 2.3 to 2.8 and then it decreases slowly with the increase of $z$ from 2.8 to 3.2 and then it increases rapidly when $z$ increases further from 3.2 to 3.5. One can see that for a given set of values of the parameters and for any angle of tapering $\psi$, the plug core radius of the two-fluid H-B model is considerably lower than that of the two-fluid Casson model. The variation of plug core radius with maximum depth of the stenosis for different values of the amplitude parameter $b$ of the time dependent radius of the artery and two-fluid H-B and Casson fluid models with $\psi=-0.1, t=45^{\circ}, \delta_{P}=\theta=0.1, B=$ $1, e=0.5, \phi=\alpha_{H}=\alpha_{C}=0.2, \omega=1, z=2.3$, and $\beta=n=0.95$ is illustrated in Figure 3. It is noted that for both of the two-fluid models, the plug core radius decreases slowly with the increase of the maximum depth of the stenosis. Figures 2 and 3 bring out the effect of angle of tapering, depth of the stenosis, and amplitude of the time dependent artery radius on the plug core radius of blood flow in a tapered artery with overlapping stenosis.

\subsection{Plug Flow Velocity}

Figure 4 shows the variation of plug flow velocity with yield stress for two-fluid H-B and Casson models and different values of $B$ and $\psi$ with $b=0.1, \beta=0.95, t=60^{\circ}, \delta_{P}=0.1$, $\omega=1, e=0.5, \phi=\alpha_{H}=\alpha_{C}=0.2, z=2.3$, and $\beta=n=0.95$. It is seen that for two-fluid H-B model, the plug flow velocity of blood decreases very slowly with the increase of yield stress 


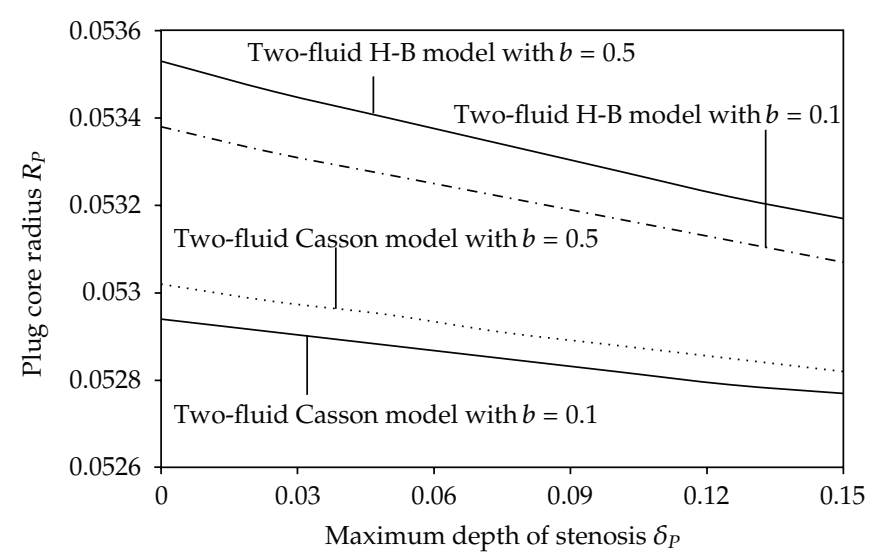

Figure 3: Variation of plug core radius with maximum depth of the stenosis for two-fluid H-B and Casson models and different values of amplitude of the artery radius with $\psi=-0.1, t=45^{\circ}, \delta_{P}=\theta=0.1, B=$ $1, e=0.5, \phi=\alpha_{H}=\alpha_{C}=0.2, \omega=1, z=2.3$, and $\beta=n=0.95$.

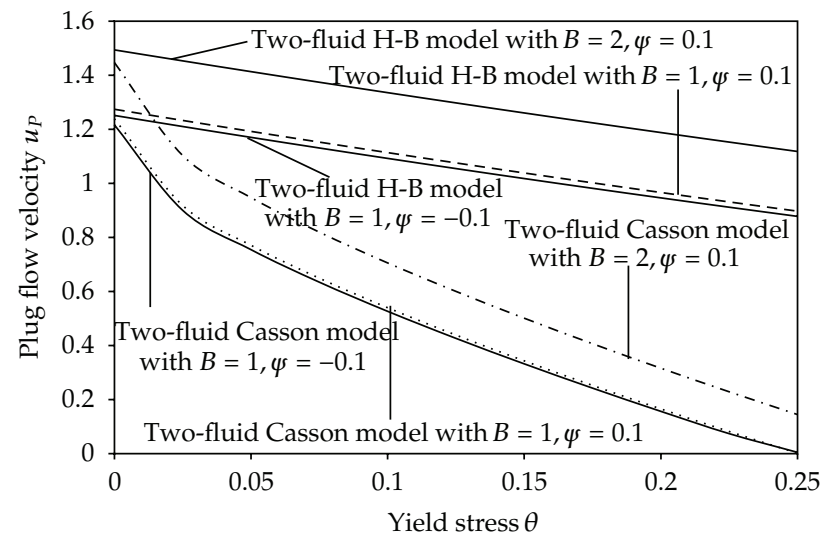

Figure 4: Variation of plug flow velocity with yield stress for two-fluid H-B and Casson fluid models and different values of $B$ and $\psi$ with $b=0.1, \beta=0.95, t=60^{\circ}, \delta_{P}=0.1, e=0.5, \phi=\alpha_{H}=\alpha_{C}=0.2, \omega=1, z=2.3$ and $\beta=n=0.95$.

$\theta$ of the blood, whereas for two-fluid Casson model, it decreases rapidly with the increase of the yield stress $\theta$ from 0 to 0.025 and then it decreases linearly when the yield stress $\theta$ increases further from 0.025 to 0.2 . It is also found that for a given set of values of all the parameters, the plug flow velocity of blood is significantly higher when it is modeled by the two-fluid model rather than by the two-fluid Casson model, which means that the plug flow velocity of blood is linear and considerably higher when it is modeled by the two-fluid H-B model. It is noted that the plug flow velocity of blood increases considerably when the body acceleration parameter $B$ increases and marginally when the angle of tapering $\psi$ of the artery increases when all the other parameters were held constant.

The plug flow velocity in a time cycle for the two-fluid H-B and Casson models and different values of the parameters $n, b$, and $\beta$ with $t=60^{\circ}, \delta_{P}=\theta=0.1, e=0.5, z=2.3$, $\phi=\alpha_{H}=\alpha_{C}=0.2, \omega=1$, and $\psi=-0.1$ is depicted in Figure 5. It is found that for the two-fluid H-B model, the plug flow velocity decreases very rapidly when the time variable $t$ increases 


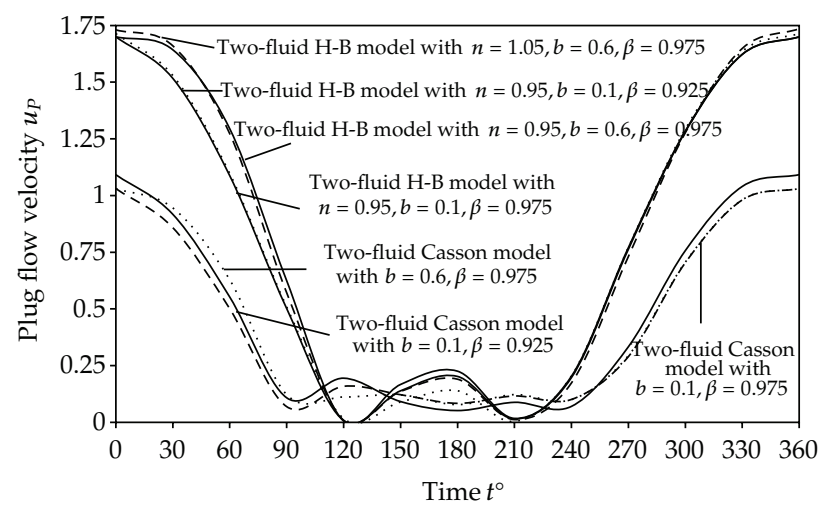

Figure 5: Variation of plug flow velocity in a time cycle for two-fluid H-B and Casson models and different values of $n, b$ and $\beta$ with $t=60^{\circ}, \delta_{P}=\theta=0.1, e=0.5, z=2.3, \omega=1, \phi=\alpha_{H}=\alpha_{C}=0.2$, and $\psi=-0.1$.

from $0^{\circ}$ to $120^{\circ}$ and then it increases slowly when $t$ increases from $120^{\circ}$ to $180^{\circ}$ and then it decreases slowly when $t$ increases from $180^{\circ}$ to $210^{\circ}$ and then it increases very rapidly when $t$ increases further from $210^{\circ}$ to $360^{\circ}$. But, for the two-fluid Casson model, its plug flow velocity decreases rapidly when the time variable $t$ increases from $0^{\circ}$ to $90^{\circ}$ and then it increases very slowly when $t$ increases from $90^{\circ}$ to $120^{\circ}$ and then it decreases very slowly when $t$ increases from $120^{\circ}$ to $180^{\circ}$ and then it increases very slowly when $t$ increases from $180^{\circ}$ to $210^{\circ}$ and then it decreases very slowly when $t$ increases from $210^{\circ}$ to $240^{\circ}$ then it increases rapidly when $t$ increases further from $240^{\circ}$ to $360^{\circ}$. It is observed that for the fixed value of the parameter $b$ of the time dependent artery radius, the plug flow velocity decreases slightly with the increase of either the power law index $n$ or the peripheral layer thickness. On the other hand, the plug flow velocity decreases considerably with the increase of the amplitude parameter $b$ of the time-dependent artery radius when all the other parameters are kept as invariables. Figures 4 and 5 bring out the effect of body acceleration, angle of tapering, peripheral layer thickness, yield stress and power law index on the plug flow velocity of blood in a tapered narrow artery with mild overlapping stenosis.

\subsection{Velocity Distribution}

Figure 6 shows the velocity distributions for two-fluid and single-fluid non-Newtonian models and Newtonian fluid model and different values of the body acceleration parameter $B$ with $n=\beta=0.95, t=210^{\circ}, b=0.1, \delta_{P}=\theta=0.1, e=0.5, \phi=\alpha=\alpha_{H}=\alpha_{C}=0.2, \omega=1, z=2.3$, and $\psi=-0.05$. It is found that the velocity is higher for fluids without yield stress than that of the fluids with yield stress. It is also seen that the highest velocity distribution is attained for the power law fluid model with $n=0.95$. The velocity distribution of the Newtonian fluid model is slightly lower than that of the power law fluid model with $n=0.95$ and the velocity distributions of the two-fluid models are considerably higher than those of the respective single-fluid models. For a given set of values of the parameters, the velocity of two-fluid H-B model is significantly higher than that of the two-fluid Casson model. It is also found that the velocity of two-fluid H-B and Casson models (or single-fluid H-B and Casson models) with body acceleration is significantly higher than those of the respective fluid models without body acceleration. It means that the presence of the body acceleration influences the velocity 


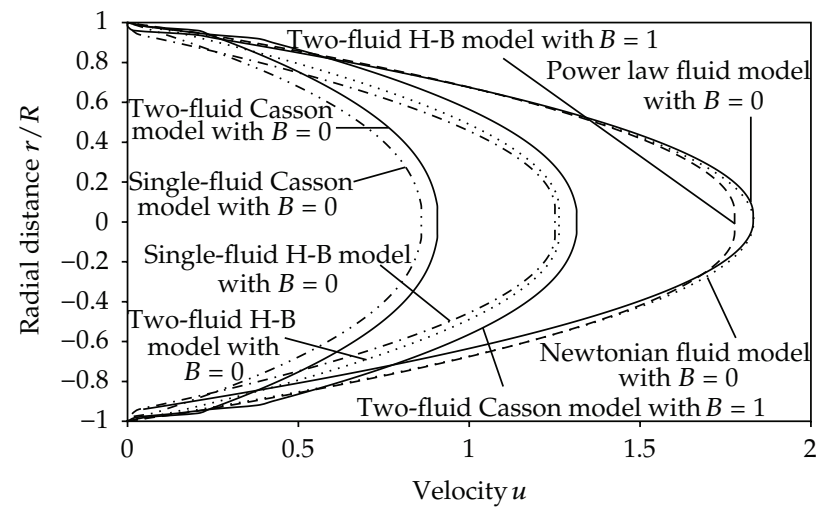

Figure 6: Velocity distribution for different fluid models and different values of $B$ with $n=\beta=0.95$, $t=210^{\circ}, b=0.1, \delta_{P}=\theta=0.1, e=0.5, \phi=\alpha=\alpha_{H}=\alpha_{C}=0.2, \omega=1, z=2.3$ and $\psi=-0.05$.

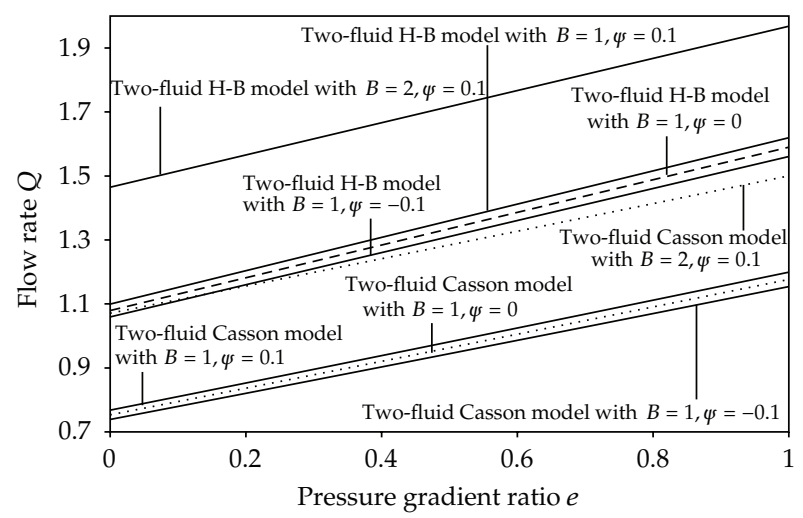

Figure 7: Variation of flow arte with pressure gradient for two-fluid H-B and Casson models and different values of $B$ and $\psi$ with $t=45^{\circ}, b=0.1, \beta=n=0.95, \delta_{P}=\theta=0.1, \omega=1, \phi=\alpha_{H}=\alpha_{C}=0.2$, and $z=2.3$.

of blood flow by increasing its magnitude significantly. It is of interest to note that the plot of the velocity distribution of the Newtonian fluid model (without body acceleration) is in good agreement with the corresponding plot in Figure 2(b) of Mekheimer and El Kot [36].

\subsection{Flow Rate}

The variation of flow rate with pressure gradient ratio for two-fluid $\mathrm{H}-\mathrm{B}$ and Casson models and different values of $B$ and $\psi$ with $t=45^{\circ}, b=0.1, \beta=n=0.95, \delta_{P}=\theta=0.1, \omega=1$, $\phi=\alpha_{H}=\alpha_{C}=0.2$, and $z=2.3$ is sketched in Figure 7. It is clear that the flow rate of blood increases linearly with the increase of the pressure gradient when blood is modeled by either of these two-fluid models. But, for a given set of values of the parameters, the flow rate of two-fluid H-B model is significantly higher than that of the two-fluid Casson model. It is also noticed that for a given set of values of the parameters, the flow rate increases with the increase of either the body acceleration $B$ or angle of tapering $\psi$. But, the increase in the flow rate is significant when the body acceleration parameter $B$ increases and marginal when the angle of tapering $\psi$ increases. Figure 8 illustrates the variation of flow rate with yield 


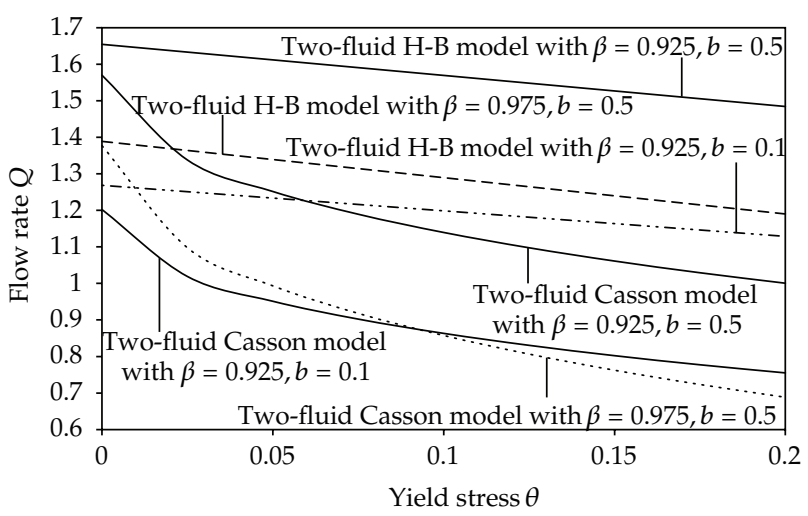

Figure 8: Variation of flow arte with yield stress for two-fluid H-B and Casson models and different values of $b$ and $\beta$ with $t=60^{\circ}, B=1, \psi=-0.1, n=0.95, \delta_{P}=0.1, \phi=\alpha=\alpha_{H}=\alpha_{C}=0.2, \omega=1$, and $z=2.3$.

stress for two-fluid H-B and Casson models and different values of $b$ and $\beta$ with $t=60^{\circ}$, $B=1, \omega=1, \psi=-0.1, n=0.95, \delta_{P}=0.1, \phi=\alpha=\alpha_{H}=\alpha_{C}=0.2$, and $z=2.3$. It is seen that the flow rate of blood decreases very slowly with the increase of the yield stress $\theta$ when blood is represented by two-fluid H-B model, whereas the flow rate of blood decreases significantly with the increase of the yield stress $\theta$ from 0 to 0.025 and then it decreases slowly with the increase of the yield stress from 0.025 to 0.2 . Also, it is observed that the flow rate of blood increases considerably with the increase of the peripheral layer thickness and the amplitude parameter $b$ of the time dependent artery radius. Figures 7 and 8 spell out the effect of peripheral layer thickness, angle of tapering, and body acceleration on the flow rate of blood when it is flowing through a tapered artery with mild constriction.

\subsection{Wall Shear Stress}

Figure 9 depicts the variation of wall shear stress with frequency ratio for two-fluid H-B and Casson models and different values of $\phi$ and $b$ with $t=60^{\circ}, B=1, \psi=-0.1, \beta=n=0.95$, $\delta_{P}=0.1, \alpha=\alpha_{H}=\alpha_{C}=0.2$, and $z=2.3$. It is found that the wall shear stress decreases slowly when the frequency ratio $\omega$ increases from 0 to 0.2 and then it decreases rapidly (nonlinearly) when the frequency ratio $\omega$ increases further from 0.2 to 1 . It is also clear that the wall shear stress in blood flow increases considerably with the increase of the amplitude $b$ of the time dependent artery radius when the lead angle is fixed. On the other hand, the wall shear stress in blood flow decreases significantly with the increase of the lead angle $\phi$ when all the other parameters were held constant. One can observe that the wall shear stress of two-fluid H-B model is slightly lower than that of the two-fluid Casson model.

\subsection{Longitudinal Impedance to Flow}

The variation of the longitudinal impedance to flow with axial distance for two-fluid H-B and Casson models and different values of $B$ and $\psi$ with $\beta=n=0.95, \theta=\delta_{P}=0.1$, $t=60^{\circ}, \phi=\alpha=\alpha_{H}=\alpha_{C}=0.2$, and $b=0.1$ is illustrated in Figure 10. It is observed that the longitudinal impedance to blood flow increases rapidly when the axial variable $z$ increases from 2 to 2.3 and then it decreases slowly with the increase of $z$ from 2.3 to 2.8 


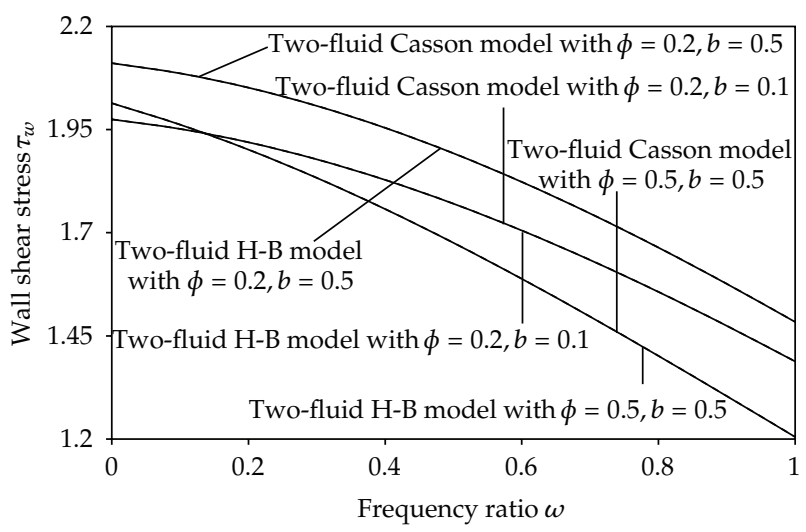

Figure 9: Variation of wall shear stress with frequency ratio for two-fluid H-B and Casson fluid models and different values of $\phi$ and $b$ with $t=60^{\circ}, B=1, \psi=-0.1, \beta=n=0.95, \delta_{P}=0.1, \alpha=\alpha_{H}=\alpha_{C}=0.2$, and $z=2.3$.

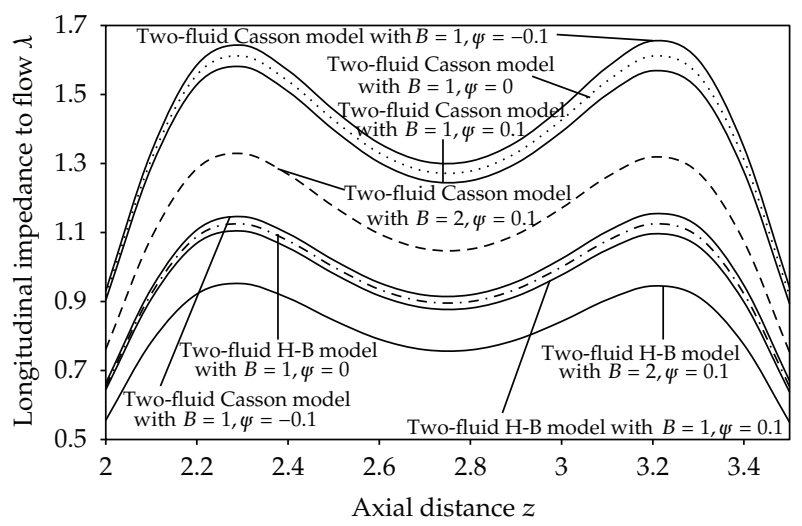

Figure 10: Variation of longitudinal impedance to flow with axial distance for two-fluid H-B and Casson models and different values of $B$ and $\psi$ with $\beta=n=0.95, \theta=\delta_{P}=0.1, \phi=\alpha=\alpha_{H}=\alpha_{C}=0.2, t=60^{\circ}$, and $b=0.1$.

and then it increases slowly when $z$ increases from 2.8 to 3.2 and then it decreases rapidly when the axial variable $z$ increases further from 3.2 to 3.5. One can notice that for a given set of values of the parameters, the longitudinal impedance to flow of the two-fluid H-B model is significantly lower than that of the two-fluid Casson model. It is also found that the longitudinal impedance of the blood flow with body acceleration is considerably lower when compared to the longitudinal impedance of the blood flow without body acceleration, meaning that the presence of body acceleration in blood flow considerably reduces the impedance to flow. It is clear that the longitudinal impedance to blood flow decreases with the increase of the angle of tapering of the artery. Figure 11 sketches the variation of longitudinal impedance to flow with maximum depth of the stenosis for two-fluid H-B and Casson models and different values of $b$ with $B=1, \psi=-0.1, t=60^{\circ}, \beta=n=0.95, \theta=\delta_{P}=0.1$, $\phi=\alpha=\alpha_{H}=\alpha_{C}=0.2$, and $b=0.1$. It is seen that the longitudinal impedance to blood flow increases slowly with the increase of the maximum depth of the stenosis $\delta_{P}$ form 0 to 0.1 and then it increases considerably when the stenosis depth $\delta_{P}$ increases further from 0.1 to 0.15 . It is also noted that the longitudinal impedance to blood flow decreases considerably with the 


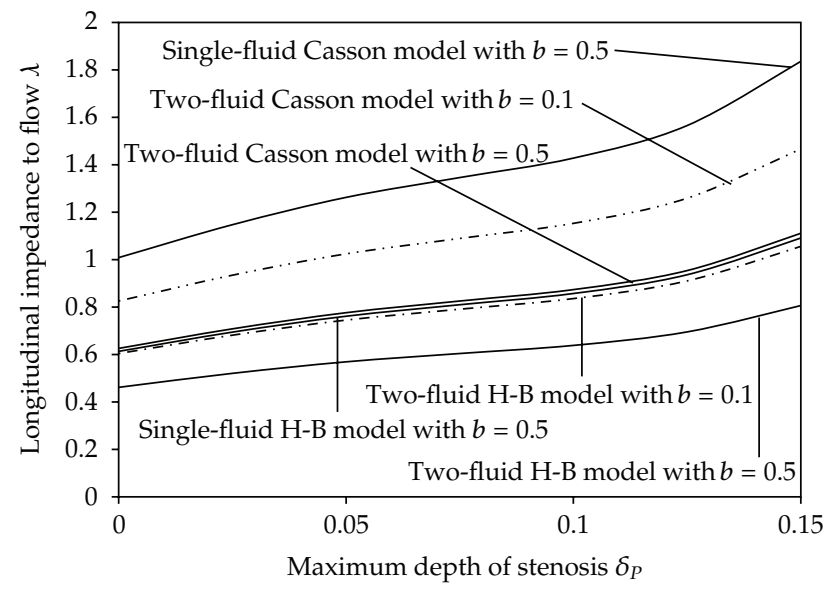

Figure 11: Variation of longitudinal impedance to flow with maximum depth of the stenosis for two-fluid $\mathrm{H}-\mathrm{B}$ and Casson models and different values of $b$ with $B=1, \psi=-0.1, t=60^{\circ}, \beta=n=0.95, \theta=\delta_{P}=0.1$, $\phi=\alpha=\alpha_{H}=\alpha_{C}=0.2$, and $b=0.1$.

increase of the amplitude parameter $b$ of the time-dependent radius of the artery. One can observe that the longitudinal impedance to flow of the two-fluid H-B model is considerably lower than that of the two-fluid Casson model. Figures 10 and 11 spell out the effects of nonNewtonian nature of blood, body acceleration, time dependent radius, and angle of tapering of the artery on the longitudinal impedance to blood flow in a constricted narrow tapered artery.

The increase in the longitudinal impedance to blood flow due to the increase in the maximum depth of the stenosis is defined as the ratio between the longitudinal impedance to flow of a fluid model for a given set of values of the parameters in an artery with stenosis and the longitudinal impedance of the same fluid model and for the same set of values of the parameters in the normal artery. The estimates of the increase in the longitudinal impedance to flow due to the increase in the maximum depth of the stenosis for two-fluid H-B and Casson models and for different angles of tapering with $B=1, t=60^{\circ}, \beta=n=0.95, \omega=1$, $e=0.5, \theta=0.1, \phi=\alpha=\alpha_{H}=\alpha_{C}=0.2, z=2.3$, and $b=0.1$ are computed in Table 1. It is observed that the estimates of the increase in the longitudinal impedance to blood flow increase slowly with the increase of the maximum depth of the stenosis and they decrease very slowly with the increase of the angle of tapering of the artery. It is also recorded that the estimates of the increase in the longitudinal impedance to flow of the two-fluid H-B model are marginally lower than those of the two-fluid Casson model.

\subsection{Some Possible Clinical Applications}

To discuss on some possible clinical applications of this study, the physiological data (for different types of arteries, their corresponding radii, and steady and pulsatile pressure gradient values) reported by Chaturani and Issac [20] are given in Table 2 and are used in this part of study. For this clinical data (given in Table 2), the estimates of the mean velocity for two-fluid H-B and Casson models and different values of $m$ and $B$ with $t=60^{\circ}, \beta=n=0.95$, $z=2.3, \theta=\delta_{P}=0.1, \phi=\alpha=\alpha_{H}=\alpha_{C}=0.2, e=0.5, \omega=1$, and $b=0.1$ are computed in Table 3 . It is noted that the mean velocity of blood decreases significantly with the increase of 
Table 1: Estimates of the increase in the longitudinal impedance to flow due to the increase in the maximum depth of the stenosis for two-fluid $\mathrm{H}-\mathrm{B}$ and Casson models and for different angles of tapering with $B=1$, $t=60^{\circ}, \beta=n=0.95, \theta=0.1, \phi=\alpha=\alpha_{H}=\alpha_{C}=0.2, z=2.3, e=0.5, \omega=1$, and $b=0.1$.

\begin{tabular}{lcccccc}
\hline \multirow{2}{*}{$\delta_{P}$} & \multicolumn{3}{c}{ Two-fluid H-B model } & \multicolumn{3}{c}{ Two-fluid Casson model } \\
& $\psi=-0.1$ & $\psi=0$ & $\psi=0.1$ & $\psi=-0.1$ & $\psi=0$ & $\psi=0.1$ \\
\hline 0.025 & 1.1398 & 1.1392 & 1.1386 & 1.1440 & 1.1433 & 1.1627 \\
0.05 & 1.3049 & 1.3034 & 1.3020 & 1.3147 & 1.3132 & 1.3354 \\
0.075 & 1.5009 & 1.4983 & 1.4957 & 1.5183 & 1.5155 & 1.5412 \\
0.1 & 1.7350 & 1.7308 & 1.7267 & 1.7626 & 1.7582 & 1.7880 \\
0.125 & 2.0165 & 2.0103 & 2.0041 & 2.0577 & 2.0511 & 2.0858 \\
0.15 & 2.3574 & 2.3483 & 2.3393 & 2.4170 & 2.4072 & 2.4480 \\
\hline
\end{tabular}

Table 2: Physiological data for different arteries.

\begin{tabular}{lcccc}
\hline Serial number & Artery & Radius $\left(\times 10^{-2} \mathrm{~m}\right)$ & $A_{0}\left(\times 10 \mathrm{Kg} \mathrm{m}^{-2} \mathrm{~s}^{-1}\right)$ & $A_{1}\left(\times 10 \mathrm{Kg} \mathrm{m}^{-2} \mathrm{~s}^{-1}\right)$ \\
\hline 1 & Aorta & 1.0 & 7.3 & 1.46 \\
2 & Femoral & 0.5 & 32.0 & 6.4 \\
3 & Carotid & 0.4 & 50.0 & 10.0 \\
4 & Coronary & 0.15 & 698.65 & 139.74 \\
5 & Arteriole & 0.008 & 2000.0 & 400 \\
\hline
\end{tabular}

Table 3: Estimates of mean velocity of two-fluid H-B and Casson models for different values of $m$ and $B$ in arteries with different radii with $t=60^{\circ}, \beta=n=0.95, \theta=\delta_{P}=0.1, \phi=\alpha=\alpha_{H}=\alpha_{C}=0.2, z=2.3, e=0.5$, $\omega=1$, and $b=0.1$.

(a) Two-fluid H-B model $\left(\times 10^{-2} \mathrm{~m} \mathrm{~s}^{-1}\right)$

\begin{tabular}{lccccccc}
\hline \multirow{2}{*}{ Serial number } & Artery type & & $B=0$ & & & \multicolumn{3}{c}{$B=2$} \\
& & $\psi=-0.1$ & $\psi=0$ & $\psi=0.1$ & $\psi=-0.1$ & $\psi=0$ & $\psi=0.1$ \\
\hline 1 & Aorta & 41.41 & 44.35 & 47.25 & 46.34 & 49.62 & 53.25 \\
2 & Femoral & 47.73 & 50.07 & 53.82 & 51.38 & 54.67 & 57.49 \\
3 & Carotid & 47.73 & 52.07 & 55.82 & 51.38 & 54.67 & 57.49 \\
4 & Coronary & 90.43 & 93.85 & 96.61 & 93.41 & 96.33 & 99.75 \\
5 & Arteriole & 0.63 & 0.72 & 0.78 & 0.69 & 0.79 & 0.91 \\
\hline
\end{tabular}

(b) Two-fluid Casson model $\left(\times 10^{-2} \mathrm{~m} \mathrm{~s}^{-1}\right)$

\begin{tabular}{lccccccc}
\hline Serial number & Artery type & & $B=0$ & & & \multicolumn{3}{c}{$B=2$} \\
& & $\psi=-0.1$ & $\psi=0$ & $\psi=0.1$ & $\psi=-0.1$ & $\psi=0$ & $\psi=0.1$ \\
\hline 1 & Aorta & 37.41 & 40.89 & 44.72 & 42.49 & 46.01 & 50.76 \\
2 & Femoral & 42.95 & 46.05 & 50.65 & 48.79 & 51.98 & 55.14 \\
3 & Carotid & 42.95 & 46.05 & 50.65 & 49.79 & 51.98 & 55.14 \\
4 & Coronary & 84.63 & 87.67 & 92.77 & 89.36 & 92.57 & 95.85 \\
5 & Arteriole & 0.52 & 0.64 & 0.77 & 0.65 & 0.77 & 0.90 \\
\hline
\end{tabular}

the artery radius except in the arterioles and it increases considerably with the increase of the angle of tapering. It is also observed that the mean velocity of blood increases significantly with the increase of the body acceleration. From Tables 3(a) and 3(b), it is recorded that the estimates mean velocity of the two-fluid $\mathrm{H}-\mathrm{B}$ model are significantly higher than those of the two-fluid Casson model. 
Table 4: Estimates of mean flow rate of two-fluid H-B and Casson models for different values of $m$ and $B$ in arteries with different radii with $t=60^{\circ}, \beta=n=0.95, \theta=\delta_{P}=0.1, \phi=\alpha=\alpha_{H}=\alpha_{C}=0.2, z=2.3$, $e=0.5, \omega=1$, and $b=0.1$.

(a) Two-fluid H-B model $\left(\times 10^{-2} \mathrm{~m} \mathrm{~s}^{-1}\right)$

\begin{tabular}{lccccccc}
\hline Serial number & Artery type & & $B=0$ & & & $B=2$ & \\
& & $\psi=-0.1$ & $\psi=0$ & $\psi=0.1$ & $\psi=-0.1$ & $\psi=0$ & $\psi=0.1$ \\
\hline 1 & Aorta & 68.16 & 72.58 & 76.57 & 74.63 & 80.28 & 86.48 \\
2 & Femoral & 15.28 & 18.23 & 21.26 & 20.54 & 26.41 & 30.27 \\
3 & Carotid & 10.69 & 12.27 & 14.72 & 13.66 & 15.64 & 18.27 \\
4 & Coronary & 4.25 & 4.89 & 5.11 & 4.57 & 4.93 & 5.28 \\
5 & Arteriole & $72.5 E-6$ & $77.2 E-6$ & $82.4 E-6$ & $74.6 E-6$ & $80.6 E-6$ & $85.6 E-6$ \\
\hline
\end{tabular}

(b) Two-fluid Casson model $\left(\times 10^{-2} \mathrm{~m} \mathrm{~s}^{-1}\right)$

\begin{tabular}{lccccccc}
\hline \multirow{2}{*}{ Serial number } & Artery type & & $B=0$ & & & $B=2$ & \\
& & $\psi=-0.1$ & $\psi=0$ & $\psi=0.1$ & $\psi=-0.1$ & $\psi=0$ & $\psi=0.1$ \\
\hline 1 & Aorta & 63.35 & 68.54 & 73.17 & 69.46 & 75.39 & 81.81 \\
2 & Femoral & 12.85 & 15.63 & 18.83 & 16.53 & 21.18 & 26.44 \\
3 & Carotid & 8.21 & 10.87 & 13.16 & 11.43 & 13.95 & 15.73 \\
4 & Coronary & 3.16 & 3.57 & 3.95 & 3.45 & 3.99 & 4.36 \\
5 & Arteriole & $66.7 E-6$ & $71.6 E-6$ & $77.2 E-6$ & $70.1 E-6$ & $75.4 E-6$ & $81.1 E-6$ \\
\hline
\end{tabular}

For the clinical data given in Table 2, the estimates of mean flow rate for the two-fluid $\mathrm{H}-\mathrm{B}$ and Casson models and different values of $m$ and $B$ with $t=60^{\circ}, \beta=n=0.95, z=2.3$, $\theta=\delta_{P}=0.1, \phi=\alpha=\alpha_{H}=\alpha_{C}=0.2, e=0.5, \omega=1$, and $b=0.1$ are computed in Table 4 . It is found that the mean flow rate of blood increases very significantly with the increase of the artery radius and it increases considerably with the increase of the angle of tapering. One can also note that the mean flow rate of blood increases significantly with the increase of the body acceleration. From Tables 4(a) and 4(b), it is observed that the estimates of the mean flow rate of the two-fluid H-B model are considerably higher than those of the two-fluid Casson model.

\section{Conclusions}

The present comparative analysis brings out several useful rheological properties of blood when it flows through narrow tapered arteries with mild overlapping time-dependent stenosis in the presence of external periodic body acceleration, treating it as (i) two-fluid H-B model and (ii) two-fluid Casson model. Some major findings of this mathematical analysis which reveal in blood flow modeling, the advantages of treating blood as two-fluid H-B model rather than two-fluid Casson model, are summarized below.

(i) The plug core radius, wall shear stress, and longitudinal impedance to flow are marginally lower for the two-fluid H-B model compared to the corresponding flow quantities of the two-fluid Casson fluid model.

(ii) The plug flow velocity, velocity distribution, and flow rate of blood are considerably higher for the two-fluid H-B fluid model than to those of the two-fluid Casson fluid model.

(iii) The estimates of the mean velocity and mean flow rate of the two-fluid H-B model are considerably higher than those of the two-fluid Casson model. On the other 
hand, the following similarities are noticed when modeling blood by either of these two models.

(iv) The plug core radius and longitudinal impedance to flow increases with the increase of the maximum depth of the stenosis.

(v) When the angle of tapering increases, the plug flow velocity and flow rate increase and the longitudinal impedance to flow decreases.

(vi) The estimates of the mean velocity and mean flow rate increase considerably with the increase of the body acceleration and this behavior is reversed when the maximum depth of the overlapping stenosis increases.

From the results discussed, one can observe that there is substantial difference between the flow quantities of two-fluid H-B model and two-fluid Casson model, and thus it is expected that the use of two-fluid H-B fluid for blood flow in diseased artery may provide better results which may be useful to physicians in predicting the effects of periodic body accelerations and maximum depth of the stenosis in the artery on the physiologically important flow quantities. Also, the results of this study may provide some useful information to surgeons to take some crucial decisions regarding the treatment of patients, whether the cardiovascular disease can be treated with medicines or should the patient undergo a surgery. Hence, it is concluded that the present study can be considered as an improvement in the mathematical modeling of blood flow in narrow tapered arteries with mild overlapping stenosis under periodic body accelerations.

\section{Nomenclature}

$\bar{r}$ : Radial distance

$r$ : Dimensionless radial distance

$\bar{z}: \quad$ Axial distance

$z$ : Dimensionless axial distance

$n$ : $\quad$ Power law index

$\bar{p}: \quad$ Pressure

$p: \quad$ Dimensionless pressure

$P: \quad$ Dimensionless pressure gradient

$\bar{Q}: \quad$ Flow rate

$Q: \quad$ Dimensionless flow rate

$\bar{R}_{0}$ : $\quad$ Radius of the normal artery

$\bar{R}(\bar{z})$ : Radius of the artery in the stenosed region

$R(z)$ : Dimensionless radius of the artery in the stenosed region

$F(\bar{t})$ : Body acceleration function

$a_{0}$ : Amplitude of the body acceleration

$\bar{R}_{P}$ : Plug core radius

$R_{P}$ : Dimensionless plug core radius

$\bar{u}_{H}$ : Axial velocity of Herschel-Bulkley fluid

$u_{H}$ : Dimensionless axial velocity of Herschel-Bulkley fluid

$\bar{u}_{C}:$ Axial velocity of Casson fluid

$u_{C}$ : Dimensionless axial velocity of Casson fluid

$\bar{A}_{0}$ : Steady component of the pressure gradient

$\bar{A}_{1}$ : Amplitude of the pulsatile component of the pressure gradient 
$\bar{L}$ : Length of the normal artery

$\bar{d}$ : Location of the stenosis

$d$ : Dimensionless location of the stenosis

$\bar{t}$ : Time

$t$ : Dimensionless time.

\section{Greek Letters}

$\Lambda:$ Dimensionless longitudinal impedance to flow

$\phi$ : Azimuthal angle

$\dot{\gamma}$ : Shear rate

$\bar{\tau}_{y}:$ Yield stress

$\theta$ : Dimensionless yield stress

$\bar{\tau}_{H}$ : Shear stress of the Herschel-Bulkley fluid

$\tau_{H}$ : Dimensionless shear stress of Herschel-Bulkley fluid

$\bar{\tau}_{C}$ : Shear stress for Casson fluid

$\tau_{C}$ : Dimensionless shear stress of Casson fluid

$\tau_{w}$ : Dimensionless wall shear stress

$\bar{\rho}_{H}$ : Density of Herschel-Bulkley fluid

$\bar{\rho}_{C}$ : Density of Casson fluid

$\bar{\mu}_{H}$ : Viscosity of Herschel-Bulkley fluid

$\bar{\mu}_{C}$ : Viscosity of the Casson fluid

$\alpha_{H}$ : Pulsatile Reynolds number of Herschel-Bulkley fluid

$\alpha_{C}$ : Pulsatile Reynolds number of Casson fluid

$\alpha$ : Pulsatile Reynolds number ratio

$\bar{\delta}_{P}$ : Depth of the stenosis in the peripheral layer region

$\delta_{P}$ : Dimensionless depth of the stenosis in the peripheral layer region

$\bar{\omega}$ : Angular frequency of the blood flow

$\phi: \quad$ Lead angle.

\section{Subscripts}

$w$ : Wall shear stress (used for $\tau$ )

$H$ : Herschel-Bulkley fluid (used for $\bar{u}, u, \bar{\tau}, \tau$ )

$C$ : Newtonian fluid (used for $\bar{u}, u, \bar{\tau}, \tau$ )

$P$ : Plug core region.

\section{Acknowledgment}

This research work was supported by the Research University Grant of Universiti Sains Malaysia, Malaysia, (RU Grant ref. no: 1001/PMATHS/811177).

\section{References}

[1] P. Chaturani and V. R. Ponnalagar Samy, "A study of non-Newtonian aspects of blood flow through stenosed arteries and its applications in arterial diseases," Biorheology, vol. 22, no. 6, pp. 521-531, 1985. 
[2] L. H. Back, Y. I. Cho, D. W. Crawford, and R. F. Cuffel, "Effect of mild atherosclerosis on flow resistance in a coronary artery casting of man," Journal of Biomechanical Engineering, vol. 106, no. 1, pp. 48-53, 1984.

[3] S. Chakravarty, P. K. Mandal, and Sarifuddin, "Effect of surface irregularities on unsteady pulsatile flow in a compliant artery," International Journal of Non-Linear Mechanics, vol. 40, no. 10, pp. 1268-1281, 2005.

[4] P. R. Johnston and D. Kilpatrick, "Mathematical modelling of flow through an irregular arterial stenosis," Journal of Biomechanics, vol. 24, no. 11, pp. 1069-1077, 1991.

[5] N. Talukder, P. E. Karayannacos, R. M. Nerem, and J. S. Vasco, "An experimental study of the fluid dynamics of multiple non-critical stenoses," Journal of Biomechanical Engineering, vol. 104, no. 2, pp. 143-147, 1982.

[6] P. Chaturani and V. Palanisamy, "Casson fluid model for pulsatile flow of blood under periodic body acceleration," Biorheology, vol. 27, no. 5, pp. 619-630, 1990.

[7] S. U. Siddiqui, N. K. Verma, S. Mishra, and R. S. Gupta, “Mathematical modelling of pulsatile flow of Casson's fluid in arterial stenosis," Applied Mathematics and Computation, vol. 210, no. 1, pp. 1-10, 2009.

[8] S. Chakravarty and P. K. Mandal, "A nonlinear two-dimensional model of blood flow in an overlapping arterial stenosis subjected to body acceleration," Mathematical and Computer Modelling, vol. 24, no. 1, pp. 43-58, 1996.

[9] D. S. Sankar, J. Goh, and A. I. M. Ismail, “FDM analysis for blood flow through stenosed tapered arteries," Boundary Value Problems, vol. 2010, Article ID 917067, 16 pages, 2010.

[10] K. C. Ang and J. Mazumdar, "Mathematical modelling of triple arterial stenoses," Australasian Physical and Engineering Sciences in Medicine, vol. 18, no. 2, pp. 89-94, 1995.

[11] S. Chakravarty, Sarifuddin, and P. K. Mandal, "Unsteady flow of a two-layer blood stream past a tapered flexible artery under stenotic conditions," Computational Methods in Applied Mathematics, vol. 4, no. 4, pp. 391-409, 2004.

[12] T. V. How and R. A. Black, "Pressure losses in non-Newtonian flow through rigid wall tapered tubes," Biorheology, vol. 24, no. 3, pp. 337-351, 1987.

[13] S. Chakravarty, P. K. Mandal, and A. Mandal, "Mathematical model of pulsatile blood flow in a distensible aortic bifurcation subject to body acceleration," International Journal of Engineering Science, vol. 38, no. 2, pp. 215-238, 2000.

[14] D. S. Sankar and A. I. M. Ismail, "Effect of periodic body acceleration in blood flow through stenosed arteries-a theoretical model," International Journal of Nonlinear Sciences and Numerical Simulation, vol. 11, no. 4, pp. 243-257, 2010.

[15] E. F. El-Shehawey, E. M. E. Elbarbary, N. A. S. Afifi, and M. Elshahed, "MHD flow of an elasticoviscous fluid under periodic body acceleration," International Journal of Mathematics and Mathematical Sciences, vol. 23, no. 11, pp. 795-799, 2000.

[16] P. Nagarani and G. Sarojamma, "Effect of body acceleration on pulsatile flow of casson fluid through a mild stenosed artery," Korea Australia Rheology Journal, vol. 20, no. 4, pp. 189-196, 2008.

[17] M. El-Shahed, "Pulsatile flow of blood through a stenosed porous medium under periodic body acceleration," Applied Mathematics and Computation, vol. 138, no. 2-3, pp. 479-488, 2003.

[18] R. Usha and K. Prema, "Pulsatile flow of particle-fluid suspension model of blood under periodic body acceleration," Zeitschrift fur Angewandte Mathematik und Physik, vol. 50, no. 2, pp. 175-192, 1999.

[19] N. Mustapha, S. Chakravarty, P. K. Mandal, and N. Amin, "Unsteady response of blood flow through a couple of irregular arterial constrictions to body acceleration," Journal of Mechanics in Medicine and Biology, vol. 8, no. 3, pp. 395-420, 2008.

[20] P. Chaturani and A. S. A. W. Isaac, "Blood flow with body acceleration forces," International Journal of Engineering Science, vol. 33, no. 12, pp. 1807-1820, 1995.

[21] D. S. Sankar, "A two-fluid model for pulsatile flow in catheterized blood vessels," International Journal of Non-Linear Mechanics, vol. 44, no. 4, pp. 337-351, 2009.

[22] C. Tu and M. Deville, "Pulsatile flow of Non-Newtonian fluids through arterial stenoses," Journal of Biomechanics, vol. 29, no. 7, pp. 899-908, 1996.

[23] G. Sarojamma and P. Nagarani, "Pulsatile flow of Casson fluid in a homogenous porous medium subject to external acceleration," International Journal of Nonlinear Differential Equations: Theory, Methods and Applications, vol. 7, pp. 50-64, 2002.

[24] P. K. Mandal, S. Chakravarty, A. Mandal, and N. Amin, "Effect of body acceleration on unsteady pulsatile flow of non-newtonian fluid through a stenosed artery," Applied Mathematics and Computation, vol. 189, no. 1, pp. 766-779, 2007. 
[25] R. K. Dash, G. Jayaraman, and K. N. Mehta, "Estimation of increased flow resistance in a narrow catheterized artery—a theoretical model," Journal of Biomechanics, vol. 29, no. 7, pp. 917-930, 1996.

[26] J. C. Misra and B. Pal, "A mathematical model for the study of the pulsatile flow of blood under an externally imposed body acceleration," Mathematical and Computer Modelling, vol. 29, no. 1, pp. 89-106, 1999.

[27] M. A. Ikbal, S. Chakravarty, K. K. L. Wong, J. Mazumdar, and P. K. Mandal, “Unsteady response of non-Newtonian blood flow through a stenosed artery in magnetic field," Journal of Computational and Applied Mathematics, vol. 230, no. 1, pp. 243-259, 2009.

[28] J. C. Misra and S. K. Pandey, "Peristaltic transport of blood in small vessels: study of a mathematical model," Computers and Mathematics with Applications, vol. 43, no. 8-9, pp. 1183-1193, 2002.

[29] D. S. Sankar and U. Lee, "Two-fluid Herschel-Bulkley model for blood flow in catheterized arteries," Journal of Mechanical Science and Technology, vol. 22, no. 5, pp. 1008-1018, 2008.

[30] V. P. Srivastava and M. Saxena, "Two-layered model of casson fluid flow through stenotic blood vessels: applications to the cardiovascular system," Journal of Biomechanics, vol. 27, no. 7, pp. 921-928, 1994.

[31] N. Iida, "Influence of plasma layer on steady blood flow in micro vessels," Japanese Journal of Applied Physics, vol. 17, pp. 203-214, 1978.

[32] D. S. Sankar and U. Lee, "Mathematical modeling of pulsatile flow of non-Newtonian fluid in stenosed arteries," Communications in Nonlinear Science and Numerical Simulation, vol. 14, no. 7, pp. 2971-2981, 2009.

[33] D. S. Sankar, "Perturbation analysis for blood flow in stenosed arteries under body acceleration," International Journal of Nonlinear Sciences and Numerical Simulation, vol. 11, no. 8, pp. 631-653, 2010.

[34] D. S. Sankar and U. Lee, "Nonlinear mathematical analysis for blood flow in a constricted artery under periodic body acceleration," Communications in Nonlinear Science and Numerical Simulation, vol. 16, no. 11, pp. 4390-4402, 2011.

[35] Z. Ismail, I. Abdullah, N. Mustapha, and N. Amin, "A power-law model of blood flow through a tapered overlapping stenosed artery," Applied Mathematics and Computation, vol. 195, no. 2, pp. 669$680,2008$.

[36] S. Kh. Mekheimer and M. A. El Kot, "Mathematical modeling of unsteady flow of Sisko fluid through an anisotropically tapered elastic arteries with time-variant overlapping stenosis," Applied Mathematical Modelling, vol. 36, no. 11, pp. 5393-5407, 2012.

[37] J. B. Shukla, R. S. Parihar, and S. P. Gupta, "Effects of peripheral layer viscosity on blood flow through the artery with mild stenosis," Bulletin of Mathematical Biology, vol. 42, no. 6, pp. 797-805, 1980. 


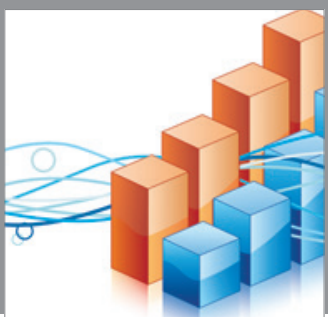

Advances in

Operations Research

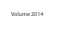

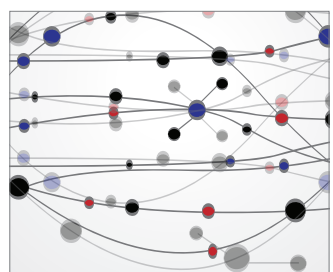

\section{The Scientific} World Journal
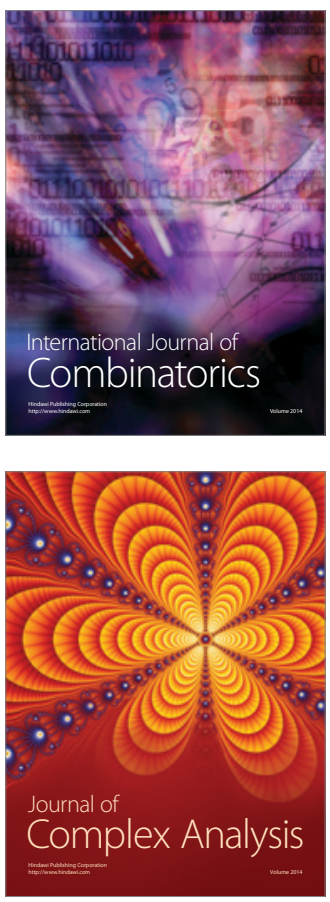

International Journal of

Mathematics and

Mathematical

Sciences
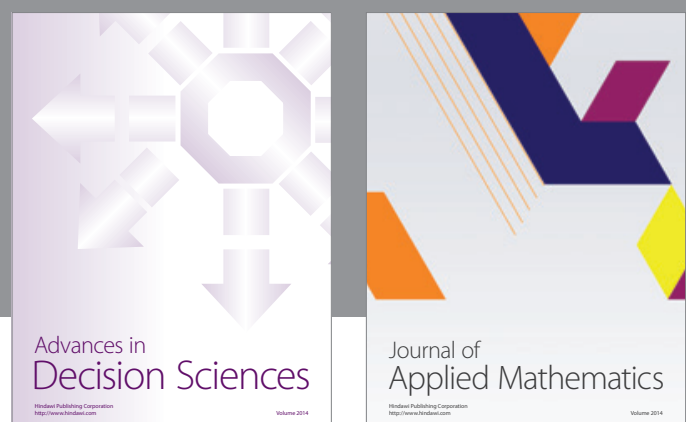

Journal of

Applied Mathematics
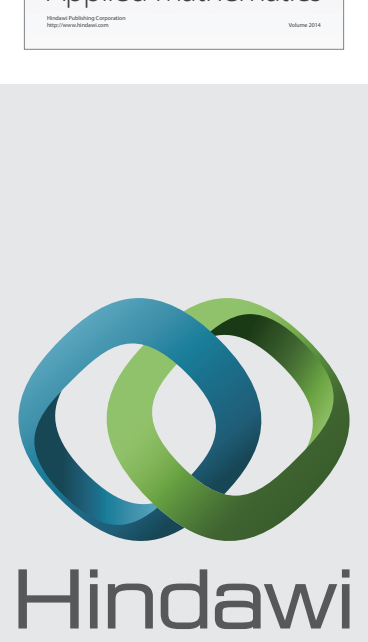

Submit your manuscripts at http://www.hindawi.com
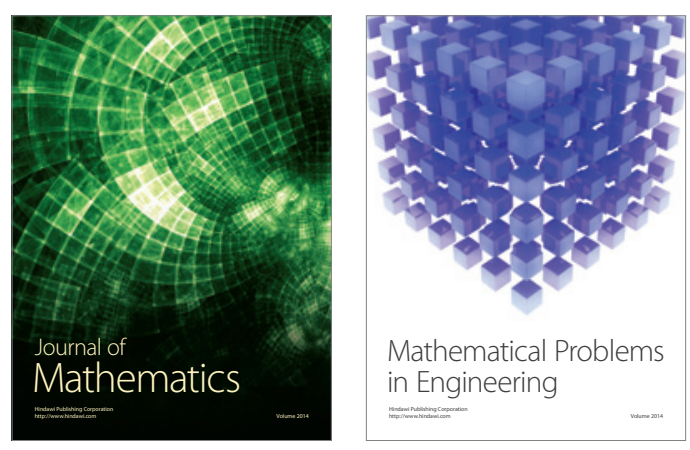

Mathematical Problems in Engineering
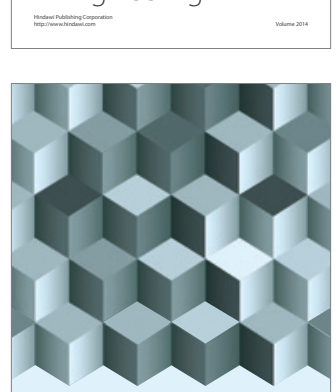

Journal of

Function Spaces
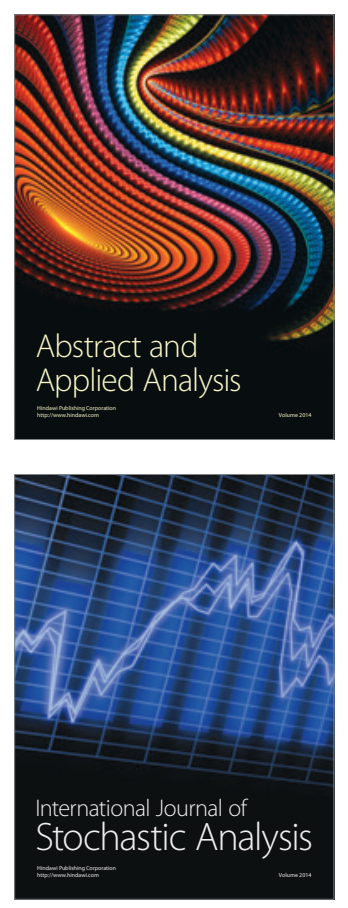

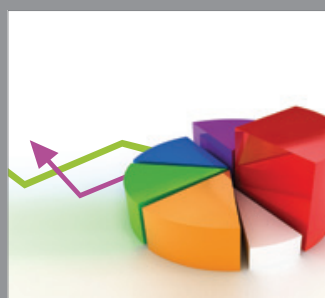

ournal of

Probability and Statistics

Promensencen
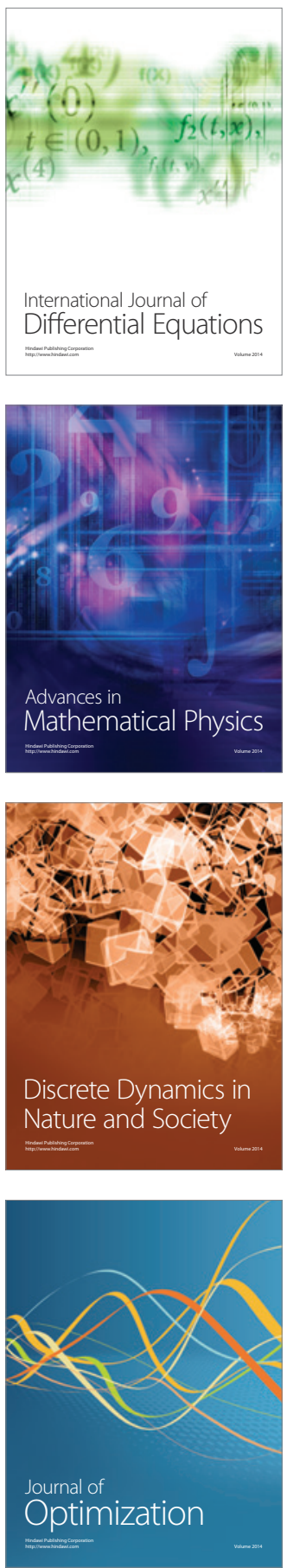